\title{
Comparing Direct and Indirect Thrust Measurements from Passively Fed and Highly Ionic Electrospray Thrusters
}

Daniel Courtney, Simon Dandavino and Herbert Shea

Microsystems for Space Technologies Laboratory, Institute of Microengineering Neuchâtel campus, School of

Engineering, École Polytechnique Fédérale de Lausanne, Rue de la Maladière 71b, 2000 Neuchâtel, Switzerland

Accepted for publication in the AIAA Journal of Propulsion and Power, 2015

This is an author generated pre-print of the final (accepted) draft manuscript. 


\title{
Comparing Direct and Indirect Thrust Measurements from Passively Fed and Highly Ionic Electrospray Thrusters
}

\author{
Daniel G. Courtney* and Simon Dandavino ${ }^{\dagger}$ and Herbert Shea ${ }^{\ddagger}$ \\ Microsystems for Space Technologies Laboratory (LMTS), \\ Ecole Polytechnique Federale de Lausanne (EPFL), Neuchatel, CH-2002, Switzerland
}

\begin{abstract}
Highly ionic beams of several hundred $\mu A$ per $\mathrm{cm}^{2}$ have been measured from porous glass ionic liquid electrospray sources fabricated using a conventional mill. The thrust output from three prototype devices, two emitting the ionic liquid EMI-Im and one emitting EMI-BF 4 , was measured directly using a precise balance. Thrusts up to $50 \mu N$ were measured when emitting EMI-Im in a bipolar, alternating potential configuration at less than $0.8 \mathrm{~W}$ input power and with propellant supplied from an internal reservoir. Measurements of mass spectra via Time of Flight spectrometry, angle resolved current distributions, ion fragmentation and energy deficits have been applied to accurately calculate thrust and mass flow rates indirectly from the same devices. For two of three cases, calculated and directly measured thrusts were in agreement to within a few $\mu N$ at input

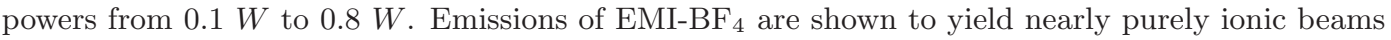
supporting high propulsive efficiencies and specific impulses, $\sim 65 \%$ and $>3200 s$ respectively at 0.5 $W$. Conversely, greater polydispersity was observed in EMI-Im emissions, contributing to reduced specific performance, $\sim 50 \%$ propulsive efficiency and $\sim 1500 \mathrm{~s}$ specific impulse at $0.5 \mathrm{~W}$.
\end{abstract}

\section{Nomenclature}

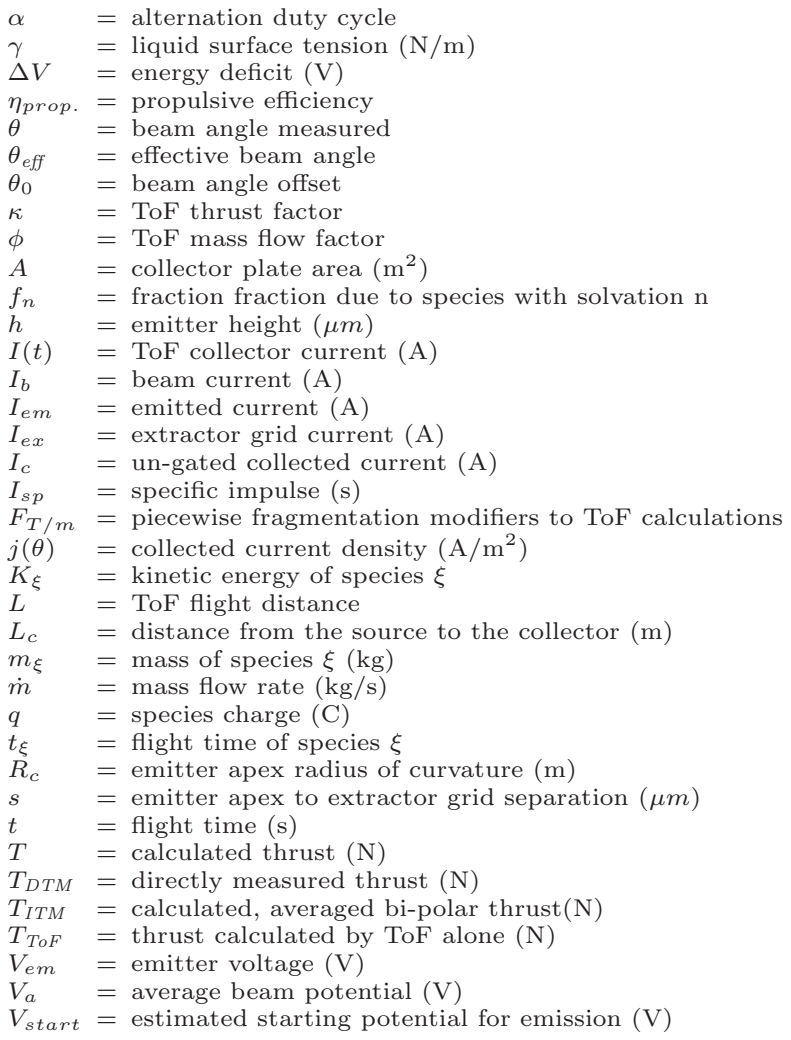

\footnotetext{
*Postdoctoral Researcher, EPFL-IMT-LMTS, AIAA Member, dcourtney@alum.mit.edu .

$\dagger$ Deputy Director, EPFL-eSpace.

¥Associate Professor, EPFL-IMT-LMTS.
}

\section{INTRODUCTION}

Interest in electrospray propulsion has seen a resurgence over the past decade with the advent of techniques for fabricating high current density emission sources[1-5] and the performance benefits identified when employing room temperature Ionic Liquids (ILs) as propellants[6-8]. IL electrosprays can yield highly ionic and monoenergetic beamlets, leading to high specific impulse and propulsive efficiency from inherently miniature emission sites (Taylor cones $[9])$. The thrust per IL emission site is small $(\sim$ 10-100 $n N$ ); hence challenges in achieving useful devices are associated with scaling up the total emission current while maintaining good specific performance. This need can be compared with, for example, plasma based propulsion systems where difficulties in maintaining high power efficiency quickly arise when attempting to miniaturize (eg. [10]). The technology is therefore well suited for small or distributable propulsion systems that require high Delta-v; of which there is a growing need/interest yet relative dearth of fully qualified systems[11, 12].

Electrospray propulsion systems targeting the Purely Ionic Regime (PIR) of emission from ILs, hereafter referred to as Ionic Liquid Ion Sources(ILIS)[7], are a particular niche sharing functional similarities yet numerous benefits compared with both colloidal [13] and Liquid Metal Ion Source LMIS[14, 15] thrusters. The potential benefits of ILIS include: i) the low surface tension of ILs leads to low operating voltages compared with LMIS, ii) ILs are liquid at room temperature and therefore may not require heating, iii) many ILs have negligible vapour pressure, enabling storage at vacuum, iv) positive and negative ion emissions can be sustained, enabling charge neutralization without a dedicated neutralizer, v) high specific impulses, in excess of $3000 s[16]$, and propulsive efficiencies nearing $90 \%[8]$ and finally, vi) the low 
flow rates inherent can be accommodated through passive feeding alone $[2,7,17]$. The later feature could greatly reduce the mass, power and volume requirements of a thruster by obviating the need for pumps, valves and propellant pressurization systems.

In this report we present a simple to fabricate form of ILIS and demonstrate, through both direct and indirect thrust measurements, that 10's of $\mu N$ thrust levels and good specific performance can be achieved from a small $(16 \mathrm{~g}, \sim 3.7 \mathrm{~cm} \times 3.7 \mathrm{~cm} \times 0.8 \mathrm{~cm})$ device wherein propellant is supplied to emission sites passively.

The low absolute thrusts $(\approx 10$ 's of $\mu N)$ yet relatively high system masses[18] of electrospray thrusters can present challenges when attempting to obtain Direct Thrust Measurements (DTM) using a dedicated balance or other force transducer, leading to few examples in the literature. In Ref. [18] precise measurements and corresponding indirect calculations are presented for a colloidal thruster. However, Ref. [16] presents the only instance of DTM from a passively fed ILIS known to the authors. That study confirmed that thrusts of $\sim 0.1$ $\mu N / \mu A$ can be achieved from arrays of ILIS and that these levels are close to those expected by extrapolation of indirect calculations based on individual emitter measurements. In section 5 we present both direct thrust measurements and a collection of probe measurements from the same devices, enabling the thrust to be calculated indirectly for comparison.

Indirect Thrust Measurements (ITM) by Time of Flight (ToF) spectrometry[19-22] are often applied to calculate expected performance. ToF measurements, which account for the spread in species charge to mass ratio within the beam, are suitable for providing a foundation for performance due to the potential diversity of particles emitted from electrospray sources. However; other factors such as energy deficits, the angular beam distribution, electrode impingements and ion fragmentation may alter the output to a measurable degree. In section 2 we arrive at performance expressions derived from a series of measurements targeting each of these factors. Subsequently in sections 5 and 6 we present and discuss a comparison between DTM and ITM as calculated using this approach.

\section{Electrospray Source Description}

Several groups have targeted high current density, passively fed ILIS which can provide 10's of $\mu N$ of thrust per $\mathrm{cm}^{2}$, or equivalently $\sim 100$ 's of $\mu \mathrm{A} / \mathrm{cm}^{2}$. Depending on the type and geometry of ILIS emitter structures, each emitter may support multiple emission sites (presumably stable Taylor cones) and yield from $\sim 0.1[7]$ to $>5 \mu A[17,23]$ of emission current. In order to generate sufficiently strong electric fields for emission while facilitating liquid transport[24], structures $\sim 100 \mu \mathrm{m}$ tall and terminated by an apex radius or capillary orifice of $O(10)$ $\mu m$ have been targeted. Such dimensions are well within the capabilities of numerous microfabrication techniques, leading to a myriad of approaches. These include silicon

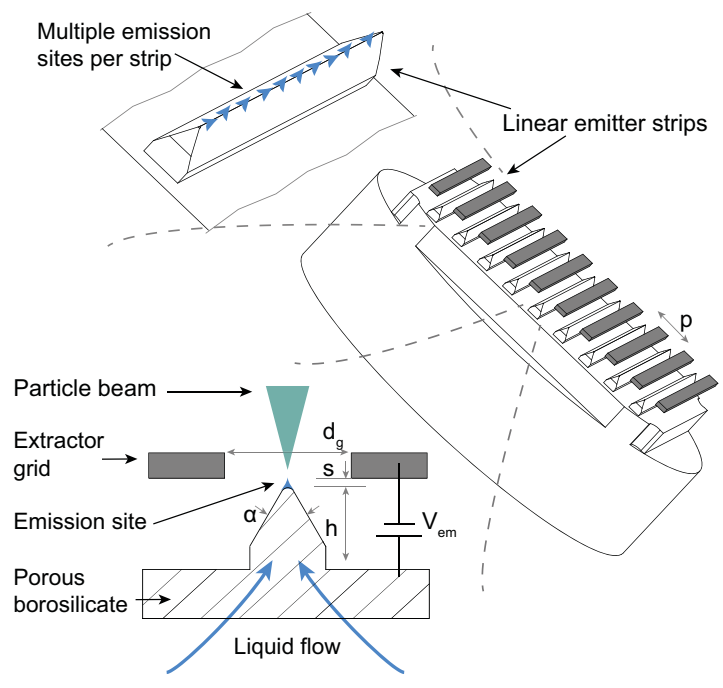

FIG. 1: Emitter strips comprising triangular prisms have been formed by milling commercially available borosilicate glass. Each emitter edge, aligned with a suspended extractor grid, is anticipated to yield a random distribution of emitter sites.

capillaries [3, 4, 25, 26], silicon tips roughened through etches or CNT growth[1,17], electrochemical etching of porous metals[16, 27], use of Rosensweig instabilities in ionic liquid ferrofluids[28] and laser ablation of porous glass[5].

Arrays of externally wetted[17] and porous $[2,5]$ ILIS have, encouragingly, been demonstrated to support a few hundred $\mu \mathrm{A}$ per $\mathrm{cm}^{2}$ of highly ionic emissions. Porous substrates are particularly advantageous in that they inherently provide a simple and integral flow path from a rear mounted and hydraulically coupled reservoir; enabling simple capillary driven and zero-g compatible passive feeding. In contrast, externally wetted arrays have, to date, been operated from a drop of liquid placed on the surface; with a continuous passive flow supply yet to be demonstrated.

We have identified and exploited two critical traits of recently demonstrated porous emitter arrays in developing a unique, low cost fabrication technique:

First, compared with precise silicon microfabrication[26], the tendency for porous emitters to achieve high and ionic currents is particularly remarkable considering the relative imprecision and range in apex radii typical of processes which have successfully been applied (eg. few to 10's of $\mu m$ in [27]). In porous materials, the Taylor cone base diameter likely scales with the characteristic length scale of the reservoir pore's Laplace pressure; leading to observations of multiple, small emission sites from each emitting structure[24]. Although these trends require further understanding, the behavior is indicative of an apparent decoupling between the fluidic transport geometry (the pores) and that determining the electrostatic field (the emitter) when using porous structures. If a shape can 
be fabricated to yield sufficiently high fields to establish emission sites localized to pores, highly ionic and high current beams have tended to follow; including at multiple points on each structure.

Second, the high conductivities and moderate dielectric constants of ILs enable emission from non-conductive glass structures[5, 29]. Furthermore, the brittle nature of porous glass enables abrasive machining with reduced risk of sealing surface pores, as could result with relatively malleable porous metals.

Figure 1 presents an overview of the emitter geometry and functional configuration applied here. In lieu of individual microfabricated emitters, a small conventional CNC mill was used to create emitting structures on commercial $1 \mathrm{~cm}$ diameter borosilicate filter discs (Duran Group Inc. P5 grade). This method is not well suited for fabricating $2 \mathrm{D}$ arrays of small emitters as the pitch would limited to the end-mill diameter. Instead we have targeted linear emitter strips comprising triangular prisms $\sim 350 \mu \mathrm{m}$ tall, $7 \mathrm{~mm}$ long and having a half-angle of $30^{\circ}$. The apex radius of curvature is not controlled but must, at least, be larger than the particles within the porous glass, typically a few $\mu m$. In the demonstration devices presented here, 9 emitter strips are fabricated per disc, spaced $0.8 \mathrm{~mm}$ apart. The milling process is detailed in section 3 and has been developed exclusively for forming porous glass structures. However, porous metal structures of similar geometry could warrant investigation if the risk of pore sealing during milling was well managed or suppressed.

As indicated in the figure we anticipate numerous emission sites to form along the sharp edge of each emitter strip. These emission sites are expected to form somewhat randomly, wherever local electric fields, pore loci and hydraulic interactions permit. A first, empirical, approximation as to the number of anticipated emission sites can be made through considering Ref. [24]. There, porous nickel emitters were positioned roughly 50 to 210 $\mu m$ below a solid stainless steel plate which served as both an extractor electrode and a simple target. After operating for 10's of minutes, numerous distinct impressions were observed on the plate and clustered around the positions of individual emitters. The impressions were assumed to be indicative of individual emission sites. Of particular relevance, when using axisymmetric emitter structures terminated by a flat plateau rather than a sharp point, numerous impressions were recorded in an often circular pattern. That observation particularly supported the notion of multiple emission sites forming from pores near the strongest electric field. In that experiment, plateau perimeters were on the order of 100 $\mu m$. Inferring that a few emission sites can therefore be sustained per $100 \mu \mathrm{m}$ of length, we anticipate that $\sim 500$ or more emission sites may be feasible from the presented devices considering the total edge length of 63 $\mathrm{mm}$; although the materials and electrostatic configurations differ significantly.

\section{INDIRECT PERFORMANCE CALCULATIONS}

Indirect thrust measurements (ITM) have been calculated with an emphasis on ToF spectrometry, complemented and refined with beam angle and Retarding Potential Analyzer (RPA) measurements. We first review the interpretation of the data acquired from each of these measurements in order to define corresponding propulsive parameters which enable functional thrust and mass flow rate calculations.

ToF measurements, where current is recorded at a detector some known distance from a fast electrostatic gate, have been applied in numerous propulsion studies [5, 19, $21,22,30,31]$ throughout the development of electrospray thrusters. Delays between the gate signal and changes in the beam current detected indicate the flight time, and thereby speed, of constituent particles within the beam. The flight time $t_{\xi}$ of particle $\xi$, relates to its mass $m_{\xi}$ and energy $q V_{a}$ through equation 1.

$$
t_{\xi}=L \sqrt{\frac{m_{\xi}}{2 q\left|V_{a}\right|}}
$$

In so far as the beam is assumed to be monoenergetic, with accelerating potential $V_{a}$, the distribution of current over flight time thus equivalently provides a distribution over mass to charge ratio $m_{\xi} / q$. The thrust and mass flow rate due to the collected species can be calculated through appropriate integrals of the ToF trace[19],

$$
\begin{gathered}
T_{\mathrm{ToF}}=-\frac{2\left|V_{a}\right|}{L} \int_{0}^{\infty} F_{T}(t) t \frac{d I(t)}{d t} d t \\
\dot{m}=-\frac{2 V_{a}}{L^{2}} \int_{0}^{\infty} F_{m}(t) t^{2} \frac{d I(t)}{d t} d t
\end{gathered}
$$

Here $T_{\text {ToF }}$ refers to the thrust as derived from ToF data and serves as the foundation for ITM in this paper. $L$ is the ToF flight tube length and $I(t)$ is the collected current as a function of flight time. The factors $F_{T}(t)$ and $F_{m}(t)$ are non-dimensional modifiers to the ToF integrand added to account for solvated ions which fragment after emission but prior to complete acceleration[32].

We are focused on IL electrospray sources operating at or near the PIR (ILIS[7]). Emitted beams are therefore primarily comprised of ions of the form $[A B]_{n} A^{+}$and $[A B]_{n} B^{-}$in the positive and negative emission modes respectively. Here $A^{+}$and $B^{-}$are the positive and negative constituent ions of the IL respectively and $n$ indicates the degree of solvation. The PIR has typically referred to emissions comprising primarily $n=0$ and $n=1$ ions, a small population of $n=2$ ions and no significant current with $n>\sim 4[7,16,33]$.

The ion population may fragment significantly in the field-free regions between the source and ToF detector. 
However these events to not alter performance and those ions are not expected to possess a significantly different speed than the parent. Hence, in the context of the Faraday cup-type detector used in this study, the speed of those detected ions alone can be interpreted to calculate the original parent mass. Conversely, ions which fragment between the emitter and extractor electrode accelerate to a greater speed than the parent ion, and therefore have a lower flight time. Without adjustment, ToF based calculations attribute intermediate masses to these ions assuming the complete energy $q V_{a}$ whereas, in actuality, signals at inter-ionic flight times are indicative of two emitted particles each with energy less than $q V_{a}$ : an ion of mass $m_{i}$ and a neutral particle of mass $m_{N}$ which is otherwise undetected. We have shown in Ref. [32] that, if specific fragmentation transitions are assumed, $F_{T}(t)$ and $F_{m}(t)$ can be simply calculated using equations 4 and 5 respectively.

$$
\begin{gathered}
F_{T}(t)=\frac{K_{i}(t)}{q V_{a}}+\left(\frac{m_{N}}{m_{i}} \frac{K_{n}(t) K_{i}(t)}{q^{2} V_{a}^{2}}\right)^{1 / 2} . \\
F_{m}(t)=\left(1+\frac{m_{N}}{m_{i}}\right) \frac{K_{i}(t)}{q V_{a}} .
\end{gathered}
$$

Energy measurements of ILIS beams have indicated that fragmentation events from solvation $n$ to $n-1$ are dominant[34]. Neglecting other transitions, the ion and neutral fractional kinetic energies are then simply related to the flight time as the functions $K_{i}(t) / V_{a}=t_{n-1}^{2} / t^{2}$ and $K_{n}(t) / q V_{a}=1-t_{n-1}^{2} / t^{2}$ respectively. Here $t_{n-1}$ refers to the ion flight time after breakup and the expressions are piecewise functions for each region $t_{n-1}<t<t_{n}$. In section 5 , we find that the effects of these adjustments on aggregate calculations are small, a few percent, yet not negligible.

It is convenient to isolate the influence of distributed $m_{i} / q$, the primary output of ToF, from the emission current and accelerating voltage in equations 2 and 3 . In this study, we consider two ionic liquids: 1-ethyl-3-methylimidazoliumbis(trifluoromethylsulfonyl)imide (EMI-Im) and 1-ethyl-

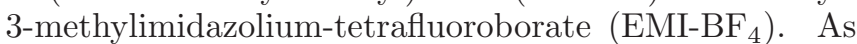
the $\mathrm{EMI}^{+}$ion is common to both liquids, we define a non-dimensional flight time $\bar{t}$ such that $t=t_{\mathrm{EMI}} \bar{t}$ where $t_{\text {EMI }}$ is the flight time expected by equation 1 for an ion of mass $m_{\text {EMI }}=111.2 D a$. Furthermore, we define a non-dimensional current $\bar{I}$ such that $I(t)=I_{c} \bar{I}(t)$ where $I_{c}$ is the current collected in the absence of a gate. It then follows that,

$$
\begin{aligned}
T_{\mathrm{ToF}} & =\kappa \sqrt{\frac{2 m_{\mathrm{EMI}}}{q}} I_{c} \sqrt{\left|V_{a}\right|}, \\
\dot{m} & =\phi \frac{m_{\mathrm{EMI}}}{q} I_{c}, \\
\kappa & =-\int_{0}^{\infty} F_{T}(\bar{t}) \bar{t} \frac{d \bar{I}(\bar{t})}{d \bar{t}} d \bar{t}, \\
\phi & =-\int_{0}^{\infty} F_{m}(\bar{t}) \bar{t}^{2} \frac{d \bar{I}(\bar{t})}{d \bar{t}} d \bar{t} .
\end{aligned}
$$

The parameters $\kappa$ and $\phi$ are non-dimensional coefficients relating thrust and mass flow rate, respectively, to the the same metrics if the beam were attributed solely to particles of mass $m_{\mathrm{EMI}}$ at the same current and voltage. Recognizing that $-d \bar{I}(\bar{t}) / d \bar{t}$ is a normalized distribution of current versus flight time and, by equation 1 , that $t$ is proportional to $\sqrt{m_{i} / q}$, we may alternatively view $\kappa$ and $\phi$ as providing relative measures of the mean $\sqrt{m_{i} / q}$ and $m_{i} / q$ respectively. Ultimately these non-dimensional parameters enable a quantitative view to the consistency in beam composition, as relevant to propulsive performance, decoupled from voltage and current.

In applying equation 7 to estimate total mass flow we have assumed an average of $\phi$ over beam angle and emitter voltage and therefore replace the ToF detector current $I_{c}$ with the total emitted current $I_{e m}$.

To calculate total thrust, consideration must be made for the spread of beam angles with respect to the thruster normal. Depending on the source type and measurement definition, ILIS beam half-angles may range from $\sim 15^{\circ}[7]$ to $40^{\circ}[35]$. To maintain relevance to propulsive parameters we define a beam angle parameter based on thrust rather than current inclusion. Consider probe measurements at an angle $\theta$ to the thruster normal. Referring to equation 6 the thrust contribution due to an element of collected current $d I_{c}=j d A$ is,

$$
d T(\theta)=\kappa \sqrt{\frac{2 m_{\mathrm{EMI}}}{q}} \sqrt{\left|V_{a}\right|} j(\theta) \cos \theta d A .
$$

Here $j$ is the current density at the sample angle $\theta$, and $d A$ refers to an elemental area at the probe collection point some $L_{c}$ from the source. $\kappa$ and $V_{a}$ may vary over angle; however (see section 6.4) we have observed these quantities to remain reasonably stable from our sources and hence consider them to be constant. The total thrust can then be estimated through integration of equation 10 over a surface $S$ enclosing the beam. Normalizing by the total beam current $I_{b}$ such that $j=\bar{j} I_{b}$, and integrating we obtain,

$$
\begin{aligned}
T & =\kappa \sqrt{\frac{2 m_{\mathrm{EMI}}}{q}} I_{b} \sqrt{\left|V_{a}\right|} \cos \theta_{\mathrm{eff}} \\
\cos \theta_{\mathrm{eff}} & \left.=\int_{S} \bar{j}(\theta) \cos \theta d A \approx \pi L_{c}^{2} \int_{0}^{\theta_{\max }} \bar{j}(\theta) \sin 2 \theta(\phi) 2\right)
\end{aligned}
$$

Intrinsically, in eqn. 11, it has been assumed that the complete beam current $\left(I_{b}\right)$ has been sampled; such that 
the surface integral of $\bar{j}$ is unity. The angle $\theta_{\text {eff }}$ is the angle of a hollow emission cone yielding the same thrust as the device under test. The approximation on the right of 12 assumes an axisymmetric beam of extent $\theta_{\max }$ and is the form ultimately applied here. In section 5 , an offset angle $\theta_{0}$ has also been calculated permitting axisymmetric beam approximations about an axis tilted with respect to the thruster.

The accelerating voltage $V_{a}$ will generally differ from the applied emitter voltage $V_{e m}$ by some deficit $\Delta V$. Reported values of $\Delta V$ range from a few volts[34, 36] to more than $100 V[4]$ depending on the source and operating conditions. Energy deficits may include intrinsic physical mechanisms, such as the ion solvation energy and Ohmic losses at the Taylor cone apex, or external influences such as series resistances upstream electrical path. Some such losses may not be constant over species mass[37]. In this study the impact of such variability was small and average fractional energy deficits were assumed for the entire spectrum. These deficits have been determined via both ToF and RPA measurements, see section 5.

Finally, it is important to acknowledge that the beam current contributing to thrust $I_{b}$ may be lower than the measured emitter current $I_{e m}$; due to interception at the extraction grid $I_{e x}$, such that $I_{b}=I_{e m}-I_{e x}$.

Considering the accumulated factors described, in equation 13, we relate the expected thrust to the readily measurable extractor voltage and beam current along and with the known mass $m_{\mathrm{EMI}}$. The specific impulse and propulsive efficiency in equations 14 and 15 respectively then follow, with the mass flow determined by equation 7 with $I_{c}$ set to $I_{e m}$.

$$
\begin{gathered}
T=\kappa \sqrt{\frac{V_{a}}{V_{e m}}} \cos \theta_{\text {eff }}\left[\sqrt{\frac{2 m_{\mathrm{EMI}}}{q}} I_{b} \sqrt{\left|V_{e m}\right|}\right] \\
I_{s p}=\frac{T}{g \dot{m}}=\frac{\kappa}{\phi} \frac{I_{b}}{I_{e m}} \sqrt{\frac{V_{a}}{V_{e m}}} \cos \theta_{\text {eff }}\left[\frac{1}{g} \sqrt{\frac{2 q\left|V_{e m}\right|}{m_{\mathrm{EMI}}}}\right] \\
\eta_{\text {prop. }}=\frac{T^{2}}{2 \dot{m} I_{e m} V_{e m}}=\left(\frac{I_{b}}{I_{e m}}\right)^{2} \frac{V_{a}}{V_{e m}} \frac{\kappa^{2}}{\phi} \cos ^{2} \theta_{\text {eff }}
\end{gathered}
$$

The specific impulse $I_{s p}$ is expressed as that which would result from pure EMI emission at the emitter voltage magnitude (in square brackets) modified by parameters due to the mass distribution, beam interception at the grid, energy deficits and angular spreading. Typical high current passively fed IL electrospray sources operate with $V_{e m} \sim 1-2 k V[2,5,22]$, corresponding to an idealized specific impulse of $\sim 4000-6000 s$ for particles of mass $m_{\mathrm{EMI}}$. However, we demonstrate in this report that lower values, $\sim$ 1000-3000 $s$, may result once all the listed parameters and both emission polarities are considered.

The constituent terms contributing to propulsive efficiency $\eta_{\text {prop. }}$ in equation 15 , are largely analogous with those considered in other electrostatic propulsion systems (eg. [38]) and are discussed in greater detail in [39] and [34]. The terms $V_{a} / V_{e m}$ and $\cos ^{2} \theta_{\text {eff }}$ are easily recognized as extraction energy and angular losses respectively while $\left(I_{b} / I_{e m}\right)^{2}$, a form of utilization efficiency, arises due to the loss of propellant and expended energy associated with accelerated particles striking the extractor grid. The quantity $\kappa^{2} / \phi$, is of particular importance for electrospray thrusters[19]. Referred to has the "polydispersive efficiency" [8], it provides a measure of the apparent power loss associated with the emission of a disperse beam, with respect to charge to mass ratios. Low polydispersive efficiencies $(<50 \%)$ can result from only a few percent of large droplets within an otherwise ionic beam[40]; greatly penalizing the power efficiency when targeting a high specific impulse through the PIR. This relatively severe loss mechanism has led to significant focus on achieving the PIR[26, 41], or more recently and with promising results, obtaining extremely small $(\sim$ few $\mathrm{kDa})$ monodisperse droplet beams[20]. In this regard, ILISs which achieve the PIR through externally wetted[7] or porous[16] electrospray emitters are a promising avenue for high efficiency, high specific impulse devices. Indeed, in [8] a relation similar to equation 15 was derived and used to motivate that total propulsive efficiencies approaching $90 \%$ may be achievable through

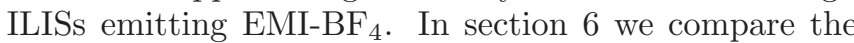
thrust calculated as per equation 11 with direct thrust measurements and apply equations 14 and 15 to estimate the specific impulse and propulsive efficiencies of three demonstration devices.

\section{SOURCE PREPARATION}

Three devices have been fabricated and tested, two wet with the IL EMI-Im and a third wet with EMI-BF 4 . These liquids were selected due to existing heritage with ILISs in the literature (for example see $[34,37]$ and $[7]$ for studies of EMI-Im and EMI-BF 4 respectively). Furthermore the heavier negative ion mass of $\mathrm{Im}^{-}(280.2 \mathrm{Da})$ versus $\mathrm{BF}_{4}^{-}(86.8 \mathrm{Da})$ and possibility of droplet (or at least very large $n$ ) emissions[37] were expected to yield higher thrusts at the expense of polydispersive efficiency. The two EMI-Im and single EMI- $\mathrm{BF}_{4}$ sources are hereafter referred to as SRC-Im-1, SRC-Im-2 and SRC-BF 4 respectively.

\subsection{Fabrication Process}

Duran Group P5 grade $1 \mathrm{~cm}$ diameter, $3 \mathrm{~mm}$ thick porous filter discs have been machined using a small table top CNC mill (Step-Four Basic 540). The forming processes is outlined in Figure 2 where dark (colored online) regions indicate material to be removed at each step. The process is comprised of the following steps: i) A square profile is defined to facilitate mounting, ii) 


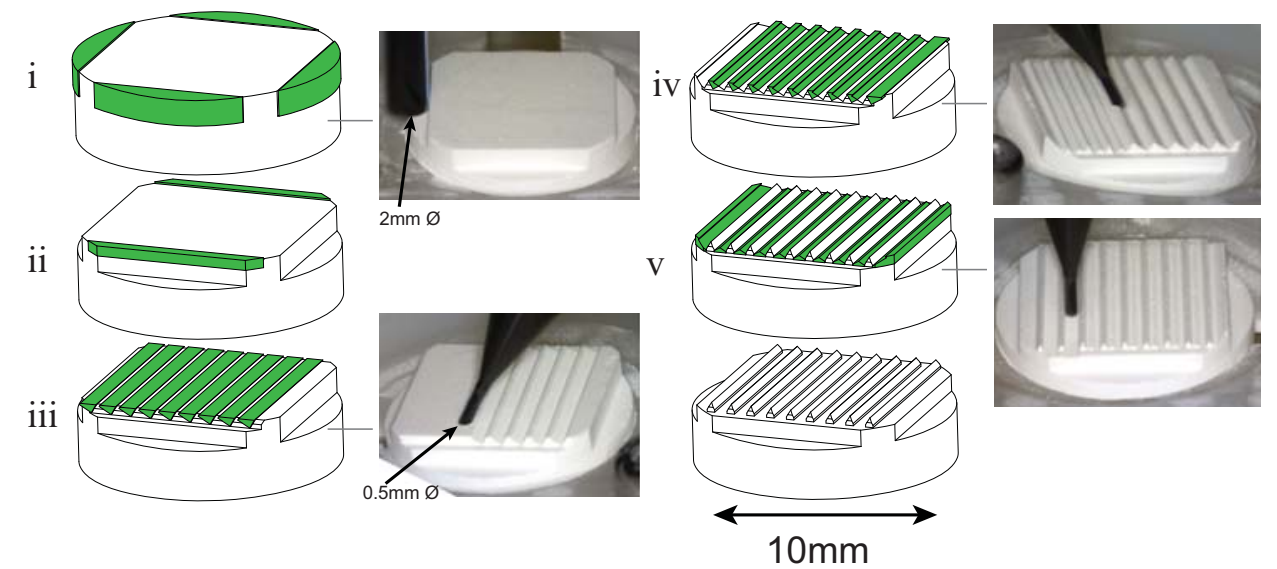

FIG. 2: Process used to machine the emitter strips used in this research from a commercially available borosilicate filter disc (Duran Group P5 grade). Coloured segments are removed at each step with a 2 or $0.5 \mathrm{~mm}$ end-mill as shown.

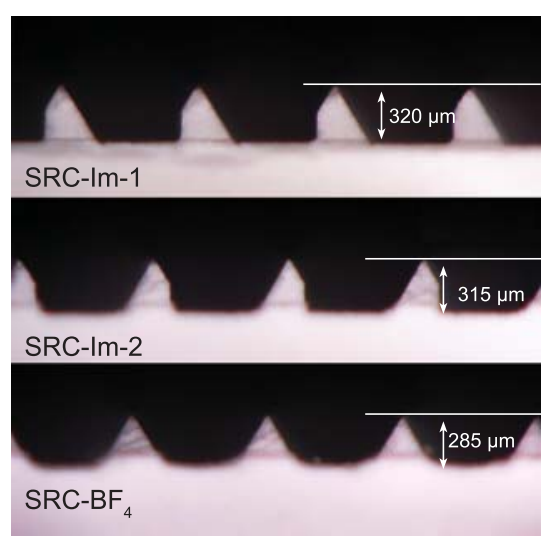

FIG. 3: Side profiles of the three devices used in this test after CNC machining. Alignment errors between steps iv and v in Fig. 2 lead to the asymmetric shapes visible in SRC-Im-1 and SRC-Im-2.

a clear out region and slight chamfer are defined to provide a buffer between the mounting flange end emitter strips iii) The sample is rotated by $30^{\circ}$ with respect to vertical and 9 cuts are made, iv) The sample is rotated to $-30^{\circ}$ with respect to vertical and offset strips are cut to fully define the emission edges, v) Finally, material between and beyond each emitter strip is removed to expose triangular prisms. Step i) and ii) through v) are made with $2 \mathrm{~mm}$ and $0.5 \mathrm{~mm}$ diameter TiAlN coated end-mills respectively. Due to the abrasive nature of borosilicates, a new $0.5 \mathrm{~mm}$ bit was required for each device. Figures 3 and 4 present photographs and a multifocal microscope image of the source emitters used in this study. The multifocal image in Figure 4 demonstrates that while low radii of curvature, a few 10's of $\mu m$, are possible with the method, an undulated ridge with a range of apex radii was typical. The asymmetric profiles in Fig. 3 for devices SRC-Im-1 and SRC-Im-2 are evidence of inaccuracies in the milling reference point between rotated configurations. However, relative to the apex radius, these asymmetries lie far from the anticipated emission site loci and the influence is not expected to be large. Generally, these inconsistencies in fabrication may be regarded as a clear shortfall when compared with the precision achievable from silicon microfabrication [17, 21, 26]; however, the tip curvature ranges are comparable with other porous sources[5, 16, 27].

\subsection{Final Assembly and Wetting}

The demonstration thruster assembly used in this study is summarized in Figure 5. Each emitter layer was stacked with a second porous borosilicate reservoir layer of larger, 16-40 $\mu \mathrm{m}$, pores (Duran Group P3 grade). These two layers are interfaced using a laser cut disc of Whatman Qualitative \# 1 filter paper; ensuring a continuous porous path between both layers. The emitter layer is supported within a PEEK mount by means of an aluminum guard ring. This flange serves two additional purposes: i) it is used as the electrical contact point with the IL and, ii) the guard ring suppresses the electric field strength at the edges of the disc, preventing spurious emissions into the extractor grid. The PEEK upper and lower mounts, and thereby the two porous discs, are then retained in contact with a mild pressure via springs. External electrical contacts to the emitter layer, via the guard ring, and the extractor grid are achieved through Pogopins soldered to a small PCB mounted on the top of the assembly. Each device was wet with IL in a two part process prior to alignment and bonding with an extractor grid. First, $90 \mu L$ of ionic liquid, which had been previously degassed for at least 1 hour under a $<10^{-5}$ mbar vacuum, was added to the the front of the device to completely fill the emitter layer. The assembly was then placed under vacuum for a further two hours to promote 


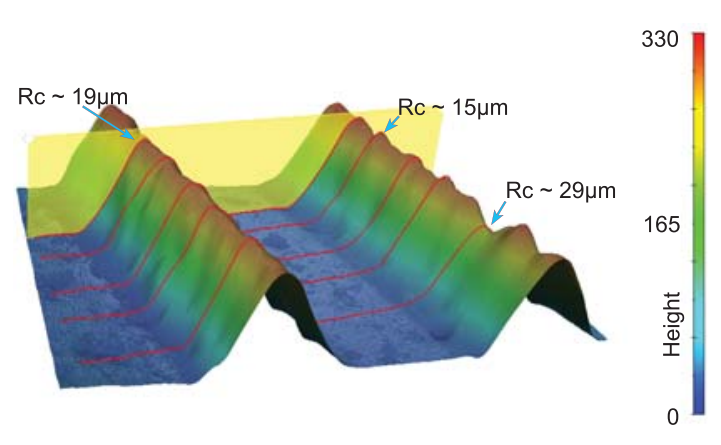

FIG. 4: Multifocal microscopic image of SRC-Im-2 indicating the typical degree of apex undulation resulting from the milling process.

filling. Finally, an additional 20 to $30 \mu L$ was added to the emitter surface in order to ensure complete saturation of the emitting layer with all excess liquid retained by the reservoir and interface layers. Liquid transport during operation was entirely passive, no external flow connections or feeding mechanisms were present.

The extractor grids comprise $350 \mu \mathrm{m}$ wide, $9 \mathrm{~mm}$ long channels laser cut from sheets of $100 \mu \mathrm{m}$ thick molybdenum. The grids were aligned using an optical microscope and held in place using layers of double sided kapton tape. This grid placement method resulted in a degree of variability in the grid position between devices. Horizontally, the alignment was typically within a few 10 s of $\mu m$ of the strip center along the length of emitter strip. However, the spacing between emitters and the grid, a relatively pertinent dimension in the context of the electric field at a given voltage, varied by 10 's of $\mu m$, see table I. In the table $s$ is the distance between the emitter edge and the base of the extractor, as indicated in Fig. 1. This preliminary design targeted a separation $s$ of zero, such that the emitting edge and extractor plate were inline; in an effort to ensure moderate starting potentials and low grid interception. However; referring to the table, the emitting edges tended to protrude into, but not through, the extractor grid for both sources emitting EMI-Im and were $105 \mu \mathrm{m}$ below the extractor on average for $\mathrm{SRC}_{-} \mathrm{BF}_{4}$.

The minimum voltage to start emission can be estimated through recognizing that the applied electrostatic pressure must overcome the liquid surface tension[38, 42] and, particularly relevant in porous devices[2], the negative Laplace pressure due to the porous reservoir. The cited relations indicate a minimum voltage which scales with the liquid surface tension $\gamma$ and length scales $f$ and $r_{p}$ for the emitter field enhancement and capillarity length scales respectively; $V_{\text {start }} \sim f \sqrt{\frac{\pi \gamma}{2 r_{p}}}[42]$. Given the transverse undulations and range of curvature radii resulting from this process and the potential for augmented field enhancement due to the liquid shape within the dielectric structure[29], accurate predictions by this relation were not expected. Nonetheless, 2D (planar) FEM

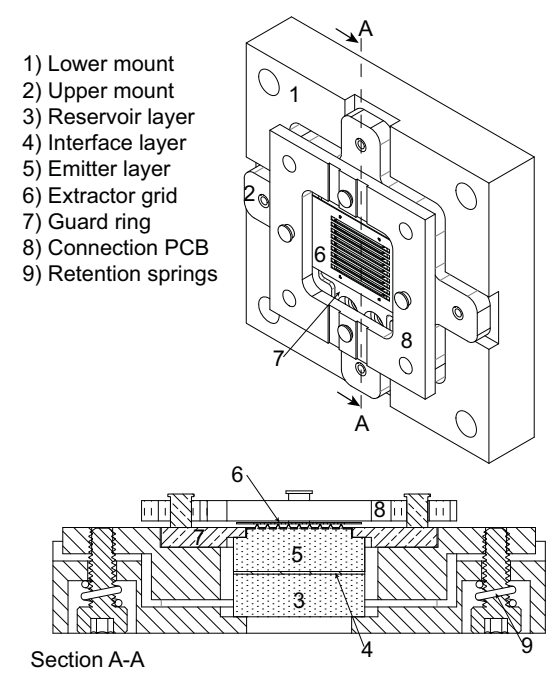

FIG. 5: Constituent components of the demonstration thrusters tested here.

TABLE I: Device Dimensions and Ionic Liquid Properties

\begin{tabular}{|c|c|c|c|c|}
\hline Device & $\begin{array}{c}h(\mu m) \\
\pm \sim 20 \mu m\end{array}$ & $s(\mu m)$ & $\begin{array}{c}\gamma(m N / m) \\
{[43]}\end{array}$ & $\begin{array}{c}V_{\text {start }}^{*}(V) \\
@ R_{c}=20 \mu m\end{array}$ \\
\hline SRC-Im-1 & 320 & $-20 \pm 20$ & 34.9 & $\sim 1550$ \\
\hline SRC-Im-2 & 315 & $-50 \pm 35$ & 34.9 & $\sim 1550$ \\
\hline $\mathrm{SRC}_{-} \mathrm{BF}_{4-1}$ & 285 & $105 \pm 30$ & 45.2 & $\sim 2100$ \\
\hline
\end{tabular}

*Predicted

simulations of the electric field were used to predict $f$ for a $20 \mu \mathrm{m}$ representative radius of curvature and the grid seperations/emitter heights listed in table I. As indicated, emissions were broadly anticipated to initiate in the vicinity of $1550 \mathrm{~V}$ and $2100 \mathrm{~V}$ for the devices wet with EMI-Im and EMI-BF 4 respectively.

The assembled devices measured $3.7 \mathrm{~cm} \times 3.7 \mathrm{~cm} \times 0.8$ $\mathrm{cm}$ and, once wet, had a typical mass of $\sim 16 \mathrm{~g}$. With IL retained by the porous reservoir, the devices can be easily handled and transported once wet; permitting tests at multiple facilities without disturbing the liquid state. The outer device dimensions could be reduced in the future to more efficiently enclose the $\sim 1 \mathrm{~cm}^{2}$ of emitting area. In particular, it should be noted that the PEEK mounting structure represented $10 \mathrm{~g}$ of the total mass and the design favoured accommodation within the test facilities over size.

\section{EXPERIMENTAL APPARATUS AND METHODS}

Measurements were acquired at both the ESA Propulsion Laboratory (EPL) within the European Space Research and Technology Centre (ESTEC), Noordwijk, the Netherlands and the Microsystems for Space Technolo- 
gies Laboratory (LMTS) at EPFL. Direct thrust measurements were made first, at ESTEC, followed by probe measurements at the LMTS.

\subsection{Direct Thrust Measurement}

The low mass and lack of fluidic connections made the demonstration thrusters suitable for DTM via a precision commercial mass balance modified for use within a vacuum facility. At the ESTEC EPL, A Mettler Toledo XP2004-S balance was installed within a $0.8 \mathrm{~m}$ diameter, $0.5 \mathrm{~m}$ tall cylindrical facility pumped by Pfeiffer TMH 261 turbo molecular pump with a Pfeiffer MVP 055-3 diaphragm forepump. With all components installed, including the wetted thruster, the base pressure was $\sim 3$ x $10^{-6}$ mbar; however, during operation at the highest currents observed for SRC-Im-1, the recorded pressure surged to up to $9 \times 10^{-5}$ mbar. Prior to testing SRCIm-2 and SRC-BF 4 an additional TMH 261 turbo pump station was added in parallel to improve the extraction rate. The peak pressure was subsequently $<6 \times 10^{-5}$ mbar when emitting.

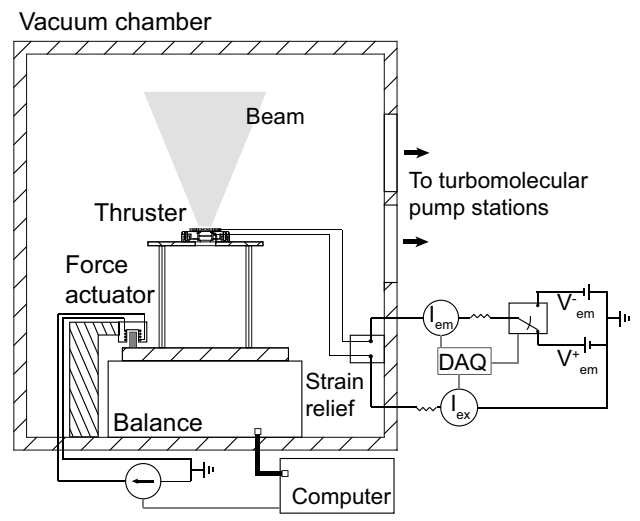

FIG. 6: Overview of the test configuration at ESTEC. The thruster was positioned to emit upwards. No fluidic connections were made to the pre-wetted and self-contained device.

The thrusters were positioned as indicated in Figure 6 ; mounted to emit upwards. Propellant wetting was performed, as described in section 3.2 , prior to installation in the facility. Hence only the emitter and extractor electrical connections were required, no fluid lines were present. The connections to high voltage vacuum feedthroughs were made via a strain relief block to reduce disturbances. The emitter voltage was supplied via FUG HCN 140-12500 and FUG HCN 35M-20000 high voltage power supplies for the positive and negative states respectively. The emitter polarity was controlled via a custom switching box controlled via a National Instruments USB-DAQ board.

The emitted and extractor (intercepted) currents were measured with optically-isolated current monitors, accurate to within $\sim 3$ and $2 \mu \mathrm{A}$ for the emitter and extractor monitors respectively. As shown explicitly in Fig. 6 each monitor included an inline high-power $100 k \Omega$ resistor along the current path to protect the monitors against short circuits. The voltage drop along these resistors has been calculated and accounted for in all data presented. That is, reported energy deficits $\Delta V$ are in excess of this known Ohmic drop.

The balance specified repeatability was $\sim 1 \mu N(0.1$ $m g$ ). In practice, see section 5 , the standard deviation in balance output was typically $\mathrm{a} \sim 2-3 \mu N$ with very low frequency (10's to 100's of $m \mathrm{~Hz}$ ) random undulations of similar order. The balance stability was therefore a significant source of measurement uncertainty during direct thrust measurements. Each thrust measurement consisted of the difference between an average over, at least, $30 \mathrm{~s}$ of thruster operation and the average of $30 \mathrm{~s}$ measurements preceding and following the measurement. The first $7 s$ of thruster activity were discarded to allow the balance and beam emissions to settle.

The balance consistency was evaluated periodically throughout the test using an electromagnetic force actuator. Here, a Keithley 2440 sourcemeter was used to drive prescribed currents through a coil to impart a consistent force, from 10 to $1000 \mu N$, while monitoring the balance output. Beginning with the unloaded balance in atmosphere, the slope of the balance output versus driving current and the drift over a $\sim 20$ minute period were recorded sequentially as a dry thruster was installed, the chamber was evacuated and, finally high voltage $(1500 \mathrm{~V})$ was applied. Once a stable configuration was reached, the sensitivity curve remained constant to within the standard error $(\sim 2 \%)$ while the change in averaged (over $30 \mathrm{~s}$ ) output was typically $<5 \mu N$ over the $\sim 20 \mathrm{~min}$ process. This feedback consistency was confirmed to persist before and after each thruster test.

\section{Potential Alternation Scheme}

Emissions from ILIS are known to decay over time[44], particulary at constant emitter polarity. These reductions can, in part, be attributed to electrochemical breakdown of IL at the liquid-conductor interface[45]. To suppress these reactions the potential is typically alternated to prevent sufficient charge accumulation at the interface for charge transfer to occur[33, 44]. Further work has indicated the efficacy of this method may be augmented when using a distal electrode[46], a scheme intrinsically implemented when using porous glass emitters as done here. The efficacy of potential alternation alone has been debated indicating that, at least, efforts should be made to balance the total charge emissions over each period[33].

In the context of our goal of comparing direct and indirect thrust measurements, potential alternation, represents an added complexity due to the possible imbalance of thrust levels between polarities. Preliminary attempts to measure thrust in a unipolar (no alternation) configuration were unreliable as the emission current tended decayed dramatically, by over $40 \%$ at times, and did 
TABLE II: Direct Thrust Measurement Setpoints

\begin{tabular}{lcc}
\hline \hline Device ID & $\sim \frac{\kappa^{+}}{\kappa^{-}}$ & $\alpha(\%)$ \\
\hline SRC-Im-1 & 0.53 & $34-36$ \\
SRC-Im-2 & 0.77 & $43-45$ \\
SRC-BF $_{4}$ & 1.0 & 50 \\
\hline \hline
\end{tabular}

not tend to fully recover even after subsequent operation with alternation (in contrast to Ref. [37]). Hence, a potential alternation scheme has been adopted. In keeping with the literature, and in lieu of accurate knowledge of the liquid-metal contact area of our device, the potential was alternated at $1 \mathrm{~Hz}$ during thrust measurements. Considering the few $s$ response time and high amplitude noise inherent with the balance, resolution of the thrust within each period was not possible. To reduce effects due to the unknown balance response when subjected to a pulsed thrust, the emitter voltage in each polarity was selected in attempt to achieve equal thrusts at any moment. The alternation duty cycle, $\alpha$ was then selected to target balanced total charge emission over each period.

Referring to equation 13, in so far as beam spreading and fractional energy deficit may be approximated as constant, the thrust from each polarity will be proportional to $\kappa\left|I_{b}\right| \sqrt{\left|V_{\text {em }}\right|}$. Despite decays, a few $(\sim 10)$ constant voltage thrust measurements were made for each device so as to estimate the ratio of proportionality factors $\kappa$ between polarities, $\kappa^{+} / \kappa^{-}$. These ratios are indicated in table II and are discussed, with the hindsight of the subsequent indirect thrust measurements in section 6 . Measurements of current versus voltage, while alternating the potential, were then used to predict the expected current imbalance at equal thrusts; thereby enabling selection of the duty cycle such that $\alpha I_{\text {em }}^{+}+(1-\alpha) I_{e m}^{-} \approx 0$. For device $\mathrm{SRC}_{-} \mathrm{BF}_{4}$, the predicted ratio of thrusts and measured current-voltage curves corresponded to well matched positive and negative outputs, leading to selection of simply matched voltages and a $50 \%$ duty cycle. This result is in keeping with previous studies where voltage alternation alone, without other means to ensure balanced charge, was shown to suppress electrochemical reactions with $\mathrm{EMI}_{-} \mathrm{BF}_{4}[44]$.

\subsection{Indirect Thrust Measurement}

The LMTS facility consists of a 6-way, ISO160 cross combined with an $0.8 \mathrm{~m}$ ISO100 extension serving as the flight tube and evacuated by a Varian V70 turbomolecular pump. The base pressure was $<3 \times 10^{-6}$ mbar, rising to $\sim 6 \times 10^{-6}$ mbar when the emission spray was active. Figure 7 provides an overview of facility as configured for ToF and beam angle measurements.

Emitter and extractor currents were monitored using the same optically-isolated transducers used at ESTEC. Similarly the emission polarity was alternated using the

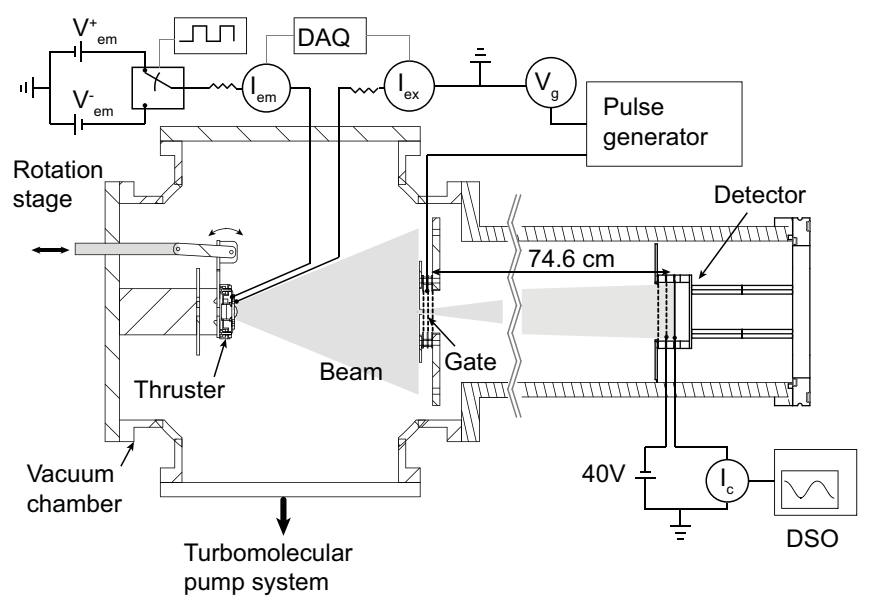

FIG. 7: Schematic of the test configuration used at LMTS as configured for ToF measurements. An aperture and baffle immediately upstream of the gate permitted $\mathrm{a} \pm 1.4^{\circ}$ half-angle beamlet to reach the detector assembly. The RPA assembly (not shown) was inserted and positioned $71 \mathrm{~mm}$ from the source when utilized.

aforementioned switching box now controlled via an Agilent 33220a arbitrary function generator. Stanford Research Systems P350 high voltage power supplies were used to control the electrostatic gate potential along with the positive and negative emitter currents.

The ToF spectrometer was positioned $13 \mathrm{~cm}$ from the source and comprised a gate assembly, flight tube and detector assembly. The flight distance from the gate electrode to the detector assembly was $74.6 \mathrm{~cm}$. The detector assembly comprised two $81 \%$ transparency stainless steel meshes upstream of a stainless steel plate, with the central mesh biased to $-40 \mathrm{~V}$ to suppress secondary electron emission from the detector plate. The detection area was $11.3 \mathrm{~cm}^{2}$.

The electrostatic gate consisted of three $81 \%$ transparency stainless steel planar meshes. The outer mesh electrodes were held at ground while the inner electrode was switched from $\pm 3000 \mathrm{~V}$ to ground at $25 \mathrm{~Hz}$ using a fast PVX-4140 high voltage switch. The detector current was converted to a voltage using a FEMTO DLPCA-100 high current amplifier, set to a gain of $10^{6} \mathrm{~V} / \mathrm{A}$ and 1 $M H z$ bandwidth. The amplified output was recorded on an LeCroy WaveSurfer 424 digital oscilloscope with $\sim 30$ trace averaging enabled to improve the recorded signal to noise ratio.

The ToF flight tube was accessed through a $6.3 \mathrm{~mm}$ diameter aperture, corresponding to a narrow $\pm 1.4^{\circ} \mathrm{ac}-$ ceptance angle. Despite the clear drawback of reduced signal intensity, the narrow acceptance angle had two benefits. Primarily, we have observed that at the 100's of $\mu A$ beam currents typical from these devices, secondary electron emission from impinged surfaces within the flight tube can become a major source of error. Spurious current signals, at times including offset currents of compa- 
rable order to the primary signal were typical until access to the flight tube was restricted. The aperture geometry was selected such that, geometrically, all particles passing into the ToF flight tube will reach the detector; that is, none are anticipated to strike the inner walls of the ToF tube upstream nor beside the collector assembly. As part of a larger baffle structure, the aperture also inhibits secondary electrons born near the flight tube entrance from reaching the high voltage gate electrode. With this system, and the aforementioned $-40 \mathrm{~V}$ screen grid upstream the detector in place, relatively clean ToF traces with low (few nA) offsets were achieved.

The thrusters were mounted on a manual rotation stage enabling angle resolved beam current and ToF measurements over $\pm 50^{\circ}$ about the central emitter strip. Beam profiles over angle were made using the ToF assembly detector with the gate electrode held to ground. The narrow acceptance angle of the ToF flight tube reduced corruption of the data due to the finite extent of the sources. Specifically, referring to equation 12 , we seek an effective angle representative of all beamlets regardless of their spatial distribution within the plane of emitting structures. The spatial spread due to long slits could present system challenges in managing the exhaust plume, but would not alter the thrust output in so far as all beamlets are considered equal. Using the aperture configuration described, the detected current contributions due to a beamlet located at the maximum extent from the center of the thruster, $\sim 4.7 \mathrm{~mm}$, were within $\sim 1^{\circ}$ of the stage angle. However; the collection solid angle for such a beamlet, as estimated using simulations with the commercial software Simion 8.1, was significantly reduced compared to emissions from the center of the device. Referring to section 2, integration of the measured current density versus angle should correspond to complete collection of the emitted beam. However; due to this reduction in transmission for distributed beamlets we have calculated, through the aforementioned simulations, that no more than $60 \%$ and as little as $40 \%$ of the emitted beam may be detected. It is important to clarify that this reduction is geometric and should not, in so far as emission characteristics are uniform over the source, correspond to a possible ambiguity in interpreting the ToF spectra.

ToF and beam angle measurements have been complimented by limited Retarding Potential Analyzer (RPA) measurements. The RPA primarily consisted of 7, stainless steel mesh grid electrodes inline with a Kimball Physics FC-72 Faraday cup detector. In keeping with previous high resolution energy measurements from ILIS $[34,36]$ the RPA design was based on the recommendations of Ref. [47]. Specifically, to ensure smooth potential gradients the retarding potential is distributed over 5 grids wherein the 3 central grids are at the retarding potential $V_{r}$ while the outer grids are maintained at $96 \%$ of $V_{r}$. A $9.5 \mathrm{~mm}$ diameter limiting aperture on the RPA's Faraday cup collector was positioned $71 \mathrm{~mm}$ from the source.

\section{RESULTS}

\subsection{Direct Thrust Measurements}

Emitted currents $\left(I_{e m}\right)$ versus the applied voltage $\left|V_{e m}\right|$ (I-V data) for each of the tested devices are shown in Figures 8 and 9. All three devices were shown to emit several hundred $\mu A$ at potentials consistent with the rough starting potential estimates in table I. These data were acquired with the potential polarity alternating at 1 $\mathrm{Hz}$ and with a $50 \%$ duty cycle. Each datum represents the mean and standard deviation (errorbars) of data collected at each polarity over a $3 s$ period. The figure insets provide representative snapshots of the raw emitted current measured at one setpoint. Short transients when switching were discarded. For both devices operated with EMI-Im, negative emission currents were lower than positive currents at a particular $\left|V_{e m}\right|$ while emission from EMI-BF 4 was relatively balanced. As shown in the Figure insets, negative polarity emitted currents tended to increase gradually across each half period for all devices, contributing to the larger standard deviations indicated in the primary figures. These I-V relationships were applied to establish the duty cycle setpoints in table II necessary to approach balanced total charge emission in tandem with equal positive and negative thrusts.

Typically a few percent of the beam was intercepted by the extraction grid, as indicated by $I_{e x}$ in the figures. Interception was greatest for SRC-Im-2, Fig. 8(b) where up to $10 \%$ of the beam was intercepted. These data are representative of the current-voltage behaviour immediately prior to DTMs. However; irreversible changes in the emission and interception currents did occur. In particular, the emission current tended to decrease gradually over the test campaign, particularly after returning to LMTS; while the current intercepted by the grid tended to decrease. The decays are discussed in more detail in section 6.3.

Raw current data and the accompanying thrust output acquired during a typical thrust measurement are shown in Figure 10, from SRC-Im-1. Figure 10(a) demonstrates how lower current, yet longer pulses per period in the negative mode were applied as per the open loop thrust and current balancing scheme. The emission current clearly decayed throughout each thrust measurement, predominantly over the first few seconds. Horizontal lines indicate the region, after a $7 s$ delay, sampled to generate representative average positive and negative currents (solid lines) and standard deviation (dashed lines) for the measurement.

In Figure 10(b) the emitter supply was off prior to and after measuring thrust, providing reference zero levels. This example demonstrates both the few $\mu N$ instabilities typical at short (few $s$ ) timescales along with a larger jump in the mean off state output before and after emission. These pre- and post-measurement zero levels were often in agreement however, as in the presented example, a discrepancy approaching $\sim 5 \mu N$ was not uncommon. 


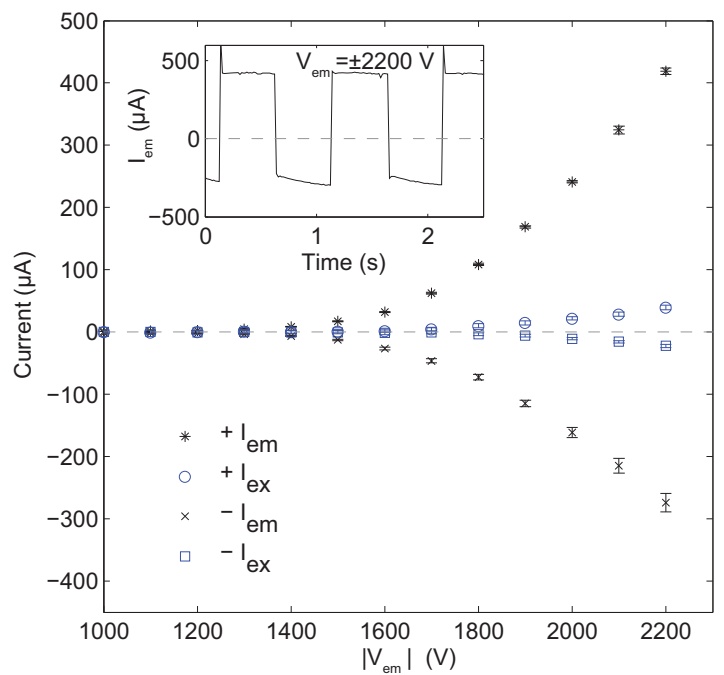

(a)SRC-Im-1

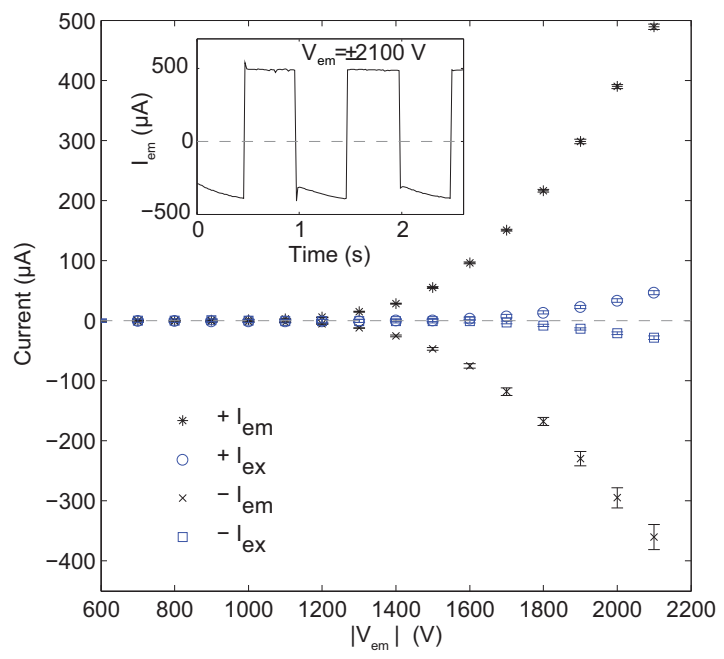

(b)SRC-Im-2

FIG. 8: Current versus voltage (I-V) characteristics for the two sources emitting the IL EMI-Im, immediately prior to direct thrust measurements.

To arrive at a single measurement, the average balance output, after a $7 s$ settling period, was compared with the average balance output for $30 \mathrm{~s}$ prior to and $30 \mathrm{~s}$ after the pulse, as indicated in the figures. Solid and dashed lines again represent the calculated means and standard deviations respectively.

In Figure 11, the thrust output from SRC-Im-2 has been plotted along with the quantity $\left\langle I_{b} \sqrt{\left|V_{e m}\right|}\right\rangle=$ $\alpha I_{b}^{+} \sqrt{V_{e m}^{+}}+(1-\alpha) I_{b}^{-} \sqrt{\left|V_{e m}^{-}\right|}$recorded during each of a sequence of 10 repeated measurements at $V_{e m}=+1997$ $V /-2007 V$. Despite the consistent emitter voltages, the beam current, and therefore $\left\langle I_{b} \sqrt{\left|V_{e m}\right|}\right\rangle$, differed between repeated trials; tending to decrease. Accordingly and consistent with equation 13 , the thrust also tended to decrease supporting correlation with this parameter,

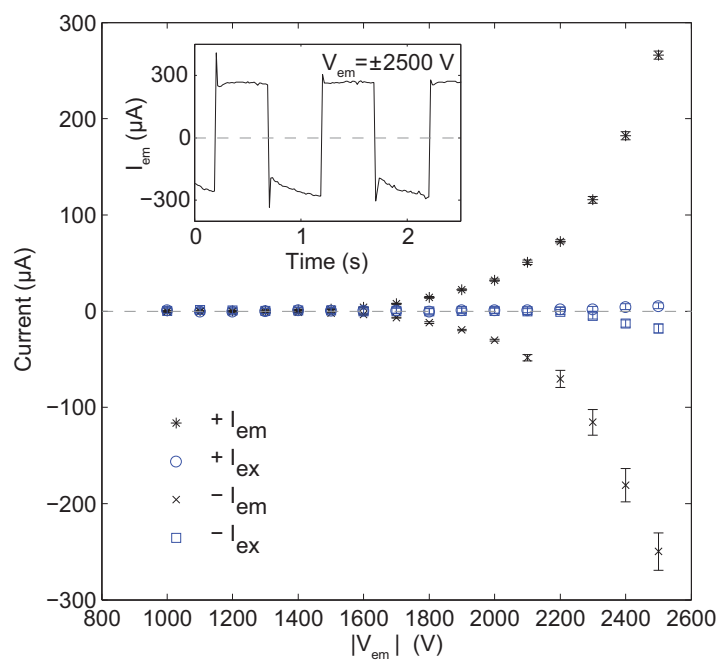

FIG. 9: Current versus voltage (I-V) characteristic for SRC$\mathrm{BF}_{4}$, immediately prior to direct thrust measurements.

see the figure inset. In section 6 , we therefore compare all direct and indirect thrust measurements as a function of $\left\langle I_{b} \sqrt{\left|V_{e m}\right|}\right\rangle$.

Scans of thrust measurements were made at set points interpolated, based on the $\mathrm{I}-\mathrm{V}$ measurements, to yield 100 to $400 \mu A$ of positive emitter current. Measurements at these set points were repeated several (3-10) times for each device, with the negative voltage and duty cycle established through table II. The summarized results, demonstrating 10's of $\mu N$ at less than $1 W$ of power for all three devices, are presented later (Figures 16 and 17) in comparison with ITM.

\subsection{Indirect Thrust Measurements}

The three devices were transported from the ESA/ESTEC EPL facilities back to the LMTS at EPFL for beam diagnostics. No modifications were made to any device, including to the liquid state. Specifically, no liquid was added or removed between tests. The devices were stored under vacuum whenever possible.

\subsubsection{Time of Flight Spectrometry}

ToF traces were acquired from each of the three test devices forming a basis for propulsive metric calculations. As described previously, the measurement setup did not permit ToF measurements while simultaneously alternating the potential. Instead all $\mathrm{ToF}$ measurements were made while emitting at constant polarity for several seconds.

Each trace was post-processed prior to determining the parameters $\kappa$ and $\phi$ by equations 8 and 9 . First the raw 


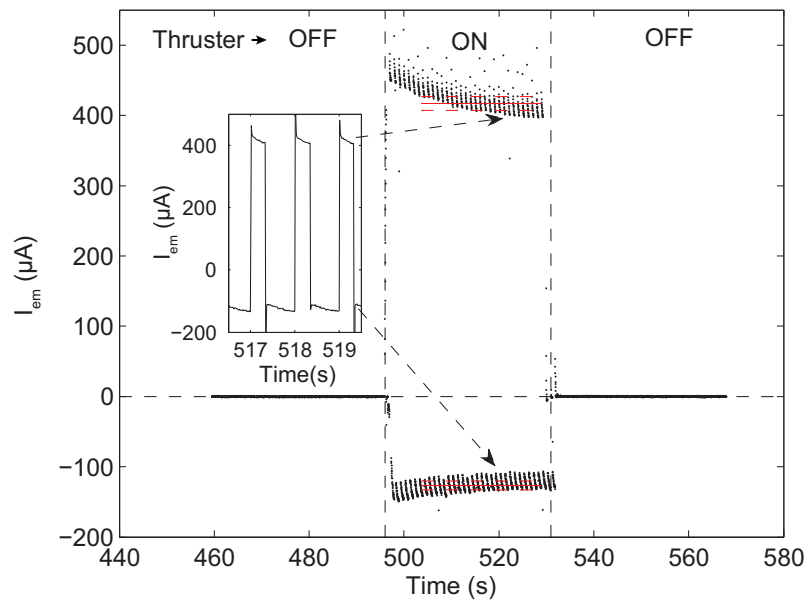

(a)Current

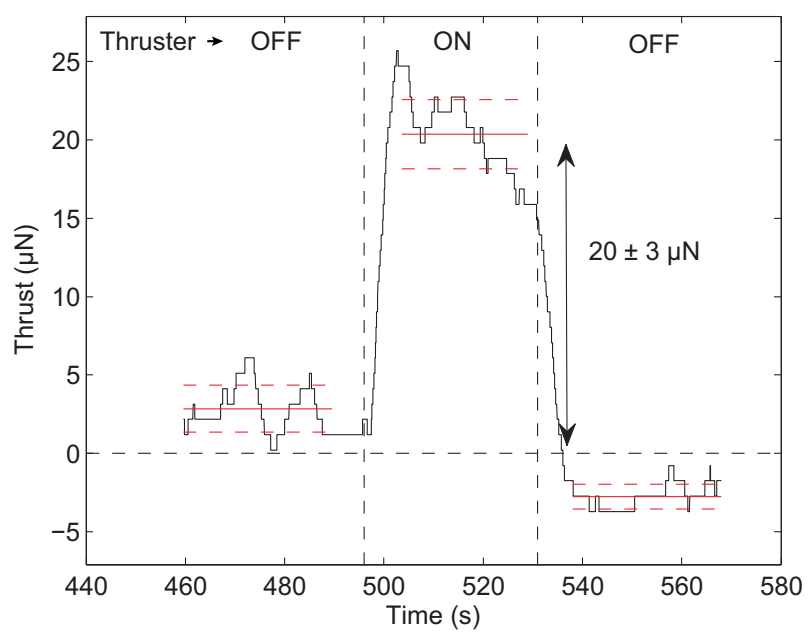

(b)Thrust

FIG. 10: Example of a typical current and corresponding thrust pulse from SRC-Im-1. The thrust measurement has been taken relative to the average of two zero level measurements recorded before and after the pulse. Solid, horizontal lines indicate the mean and standard deviation of data retained as representative of each region.

data was filtered using a Savitzky-Golay filter, as has been previously applied to ToF processing[20]. Any offset in output from the current amplifier, typically 1-2 $n A$, was then subtracted. Although modified by activating the source, this residual current was deemed spurious as it was confirmed to persist over the full $20 \mathrm{~ms}$ halfperiod of the gate signal. Finally the current trace was normalized by an average inlet current prior to the the first species flight time.

The mean shift in beam energy between the measured and expected location of ion peaks was determined through a gaussian fit to the derivative of the trace near the ion flight times anticipated by equation 1 for the $n=0$ and $n=1$ species. The edge was then taken as being $0.22 \mu \mathrm{s}$ from each peak, corresponding to half the expected decay time of the amplifier from 95 to $5 \%$ at 1

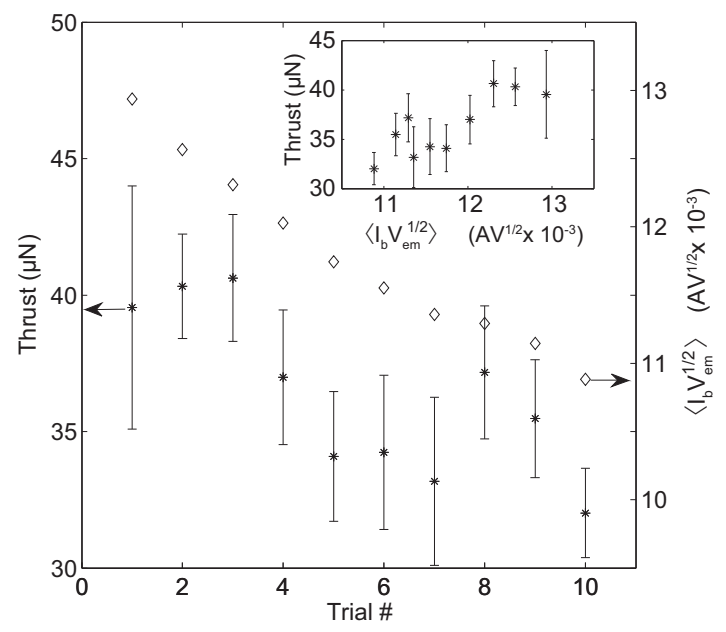

FIG. 11: Repeated thrust measurements at $+1997 \mathrm{~V}$ and $-2001 V$ and a $43 \%$ duty cycle from device EPFL4. The thruster output as reflected by both the quantity $\left\langle I_{b} \sqrt{\left|V_{e m}\right|}\right\rangle$ and the measured thrust tended to decrease over the sequence of measurements.

$M H z$ bandwidth. Voltage deficits $\Delta V_{\text {ToF }}$ for $n=0$ and $n=1$ species were taken as the difference between $V_{e m}$, after correction for the inline sensing circuit resistance, and the equivalent voltage consistent with each edge by equation 1. The average of these two deficits was then taken to estimate $V_{a}$ and used to calculate the flight time expected for ions of mass $m_{\mathrm{EMI}}$, enabling a normalized $\bar{t}$ to be calculated and applied in equations 8 and 9 for $\kappa$ and $\phi$ respectively.

ToF measurements were made at $\pm 10^{\circ}$ to $30^{\circ}$ of rotation in addition to aligned $0^{\circ}$ measurements. However; as detailed in section 6.4, trends versus neither angle nor voltage were determined to a level of confidence clearly beyond the repeatability at consistent setpoints. Instead the derived metrics presented here are an average over all measurement conditions (voltage, beam angle) per polarity.

Examples of ToF measurements for each device, at zero degree rotation, are given in Figures 12(a) though 12(b). The traces are presented as a function of the calculated species mass after correcting for energy deficits and assuming singularly charged ions. Vertical lines indicate locations of the known $n=0, n=1$ and $n=2$ ion masses; showing good agreement in the first two cases. Highly ionic emissions were achieved in all cases with most current corresponding to $n=0$ and $n=1$ species; however, a high $n$ tail extending to several $k D a$ was particularly evident at negative $V_{e m}$ from EMI-Im in Figs $12(\mathrm{a})$ and 12(b). The current contributions due to each ion species, taken as the change in fractional current between each calculated drop location, are given in table III.

Average voltage deficits for the $n=0$ and $n=1$ peaks are also provided in table III. In all instances, a higher $\Delta V$ was found for the $n=0$ ion compared with $n=1$, 


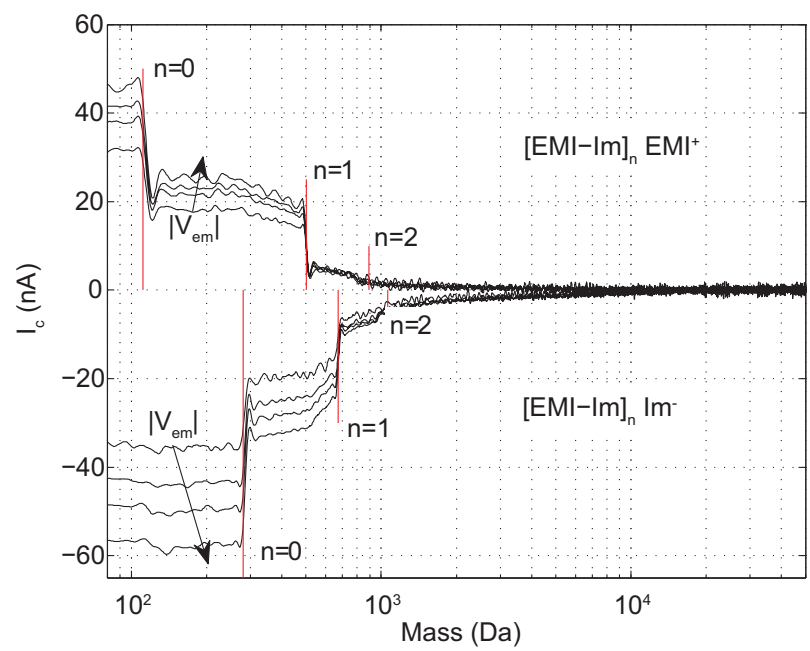

(a)SRC-Im-1: $\left|V_{e m}\right|$ increasing from $\pm 2000 \mathrm{~V}$ to $2300 \mathrm{~V}$ in $100 \mathrm{~V}$ steps.

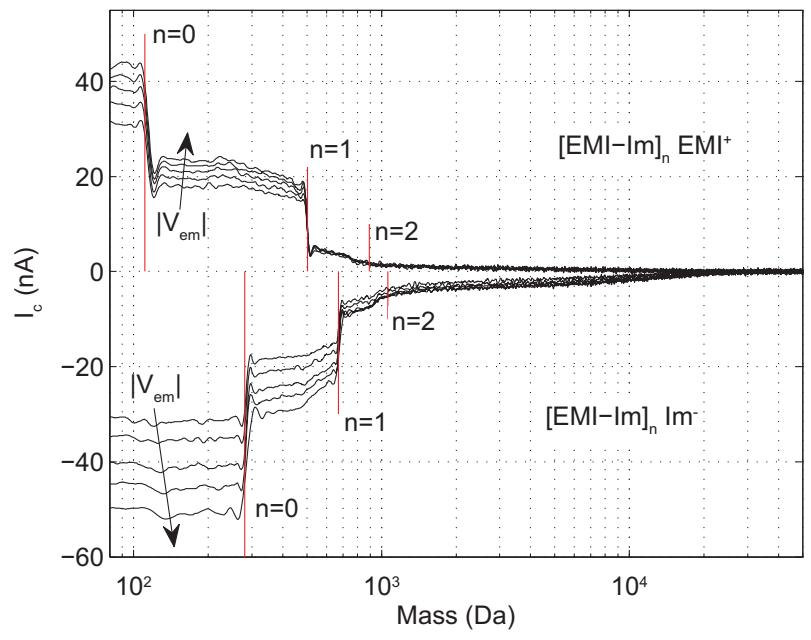

(b)SRC-Im-2: $\left|V_{\text {em }}\right|$ increasing from $\pm 1800 \mathrm{~V}$ to $2200 \mathrm{~V}$ in $100 \mathrm{~V}$ steps.

FIG. 12: ToF traces when emitting EMI-Im, a tail of large mass to charge ratio species was particularly evident for negative polarity emissions. Sloped signals between primary drops are indications of fragmentation within the emitter to extractor gap.

and lower deficits were observed at negative polarities for all devices. The fractional deficit of EMI-Im devices was, on average, less than $3 \%$ while for $\mathrm{SRC}_{-} \mathrm{BF}_{4}$ larger reductions, in excess of $7 \%$ were observed. These losses are relatively large compared with published results indicating only a few $V$ of deficit[34, 36]. In table IV the average fractional $n=0$ and $n=1 \Delta V$ are listed for each polarity facilitating inclusion in equations 13 through 15 .

The non-zero slopes between discrete ion masses can be attributed to fragmentation while accelerating between the emitter to extractor grid, where solvated $(n \geq 1)$ ions breakup into an ion and neutral component prior to complete acceleration[34, 48]. In full beam ToF where a large detector collects the entire beam, similar drops just

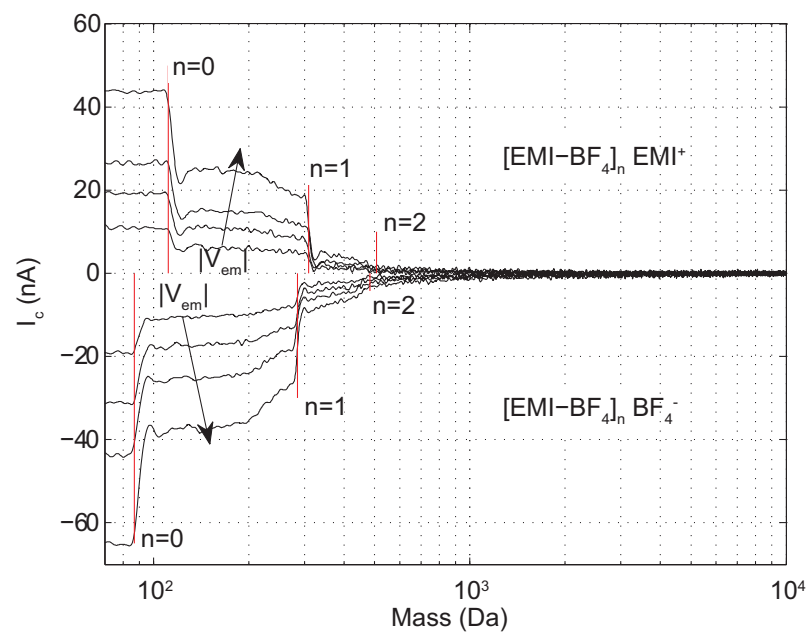

FIG. 13: $\mathrm{SRC}_{-\mathrm{BF}_{4}}:\left|V_{\text {em }}\right|$ increasing from $\pm 2100 \mathrm{~V}$ to 2400 $V$ in $100 V$ steps. Compared with Figure 12, a relatively small population of high mass to charge ratio species was observed.

after each nominal flight time may manifest due to the spread in axial velocity[48]; however, here we only sample a small portion of the beam passing within the $1.4^{\circ}$ half angle acceptance cone of the limiting aperture. Molecular dynamics simulations of fragmentation events[49] have indicated that breakup can be induced by both the ion's thermal energy and an applied electric field; and that the degree to which each process is dominant can differ between ion solvation number. Slopes in the ToF traces between the $n=1$ to $n=2$ expected masses, particularly for cations in Fig. 12, are relatively consistent despite increasing emitter voltages. If ion breakup were dictated solely by a fixed time constant, a reduction in the degree of fragmentation would be expected at higher voltages as ions are more quickly swept out of the acceleration region. However; a corresponding increase in fragmentation due to higher external fields may counter this effect. Meanwhile; the relatively shallow slopes immediately after discrete steps in the trace, particularly $n=0$, indicate ions have moved beyond the immediate vicinity of the emission site, where fields are strongest, before breaking up.

While these observations thus imply further investigation is needed to describe ion fragmentation more completely, RPA measurements presented in section 5.2.3 confirm kinetic energy deficits over a range consistent with this phenomenon. We have therefore applied fragmentation correction factors, equations 4 and 5, attributing non-zero slopes to fragmentation events alone; $n=2 \rightarrow 1$ for $t_{1}<t<t_{2}$ and $n=1 \rightarrow 0$ for $t_{0}<t<t_{1}$. The aggregate influence of these corrections (increases) on the metrics $\kappa$ and $\phi$ have tended to be small, up to $\sim 3 \%$ and $5 \%$ for the thrust and mass flow rate respectively. However; the presented values have nonetheless been adjusted to include compensation for fragmentation for completeness. 
TABLE III: Beam Composition and Energy Deficits*

\begin{tabular}{|c|c|c|c|c|c|}
\hline Device & Ion & $\mathrm{n}$ & $f_{n}(\%)$ & $\Delta V_{\mathrm{ToF}}(V)$ & $\frac{\Delta V_{\mathrm{ToF}}}{V_{e m}}(\%)$ \\
\hline \multirow{4}{*}{ SRC-Im-1 } & $\mathrm{EMI}^{+}[\mathrm{EMI}-\mathrm{Im}]_{n}$ & 0 & $40 \pm 3$ & $55 \pm 20$ & $2.7 \pm 0.8$ \\
\hline & & 1 & $47 \pm 3$ & $30 \pm 15$ & $1.6 \pm 0.7$ \\
\hline & & 2 & $9.5 \pm 2$ & - & - \\
\hline & & $\geq 3$ & $4 \pm 1.5$ & - & - \\
\hline \multirow[t]{4}{*}{ SRC-Im-1 } & $\mathrm{Im}^{-}[\mathrm{EMI}-\mathrm{Im}]_{n}$ & 0 & $40 \pm 3$ & $26 \pm 4$ & $1.2 \pm 0.1$ \\
\hline & & 1 & $40 \pm 2$ & $13 \pm 3$ & $0.6 \pm 0.1$ \\
\hline & & 2 & $10 \pm 2$ & - & - \\
\hline & & $\geq 3$ & $10 \pm 2$ & - & - \\
\hline \multirow[t]{4}{*}{ SRC-Im-2 } & $\mathrm{EMI}^{+}[\mathrm{EMI}-\mathrm{Im}]_{n}$ & 0 & $44 \pm 3$ & $70 \pm 45$ & $2.3 \pm 0.6$ \\
\hline & & 1 & $43 \pm 3$ & $55 \pm 40$ & $1.3 \pm 0.5$ \\
\hline & & 2 & $9.5 \pm 3$ & - & - \\
\hline & & $\geq 3$ & $3.5 \pm 2$ & - & - \\
\hline \multirow[t]{4}{*}{ SRC-Im-2 } & $\mathrm{Im}^{-}[\mathrm{EMI}-\mathrm{Im}]_{n}$ & 0 & $40 \pm 4$ & $23 \pm 4$ & $1.2 \pm 0.2$ \\
\hline & & 1 & $40 \pm 4$ & $14 \pm 4$ & $0.7 \pm 0.1$ \\
\hline & & 2 & $9 \pm 3$ & - & - \\
\hline & & $\geq 3$ & $10 \pm 3$ & - & - \\
\hline \multirow[t]{4}{*}{$\mathrm{SRC}_{-} \mathrm{BF}_{4}$} & $\mathrm{EMI}^{+}\left[\mathrm{EMI}^{\left.-\mathrm{BF}_{4}\right]_{n}}\right.$ & 0 & $42 \pm 2$ & $160 \pm 25$ & $7.2 \pm 1.1$ \\
\hline & & 1 & $47 \pm 3$ & $140 \pm 15$ & $6.2 \pm 0.7$ \\
\hline & & 2 & $8.5 \pm 2$ & - & - \\
\hline & & $\geq 3$ & $3.5 \pm 2$ & - & - \\
\hline \multirow[t]{4}{*}{$\mathrm{SRC}_{-} \mathrm{BF}_{4}$} & $\mathrm{BF}_{4}^{-}\left[\mathrm{EMI}^{\left.-\mathrm{BF}_{4}\right]_{n}}\right.$ & 0 & $44 \pm 2$ & $97 \pm 9$ & $4.2 \pm 0.4$ \\
\hline & & 1 & $44 \pm 3$ & $61 \pm 6$ & $2.7 \pm 0.3$ \\
\hline & & 2 & $8.5 \pm 2$ & - & - \\
\hline & & $\geq 3$ & $4.5 \pm 1.5$ & - & - \\
\hline
\end{tabular}

*Uncertainties indicate the standard deviation over 19 or more measurements

Average propulsive parameters $\kappa, \phi$, and the resultant polydispersive efficiency $\left(\kappa^{2} / \phi\right)$ are given in table IV. Of particular relevance to our goal of comparing direct and indirect thrusts, the standard deviation of the parameter $\kappa$ was, at most, $\sim 7 \%$ despite the inelegance of averaging values over all voltage and beam angles measurement conditions. Relatively large fluctuations in $\phi$ contributed to the large propagated errors in polydispersive efficiency reported. Regardless, the comparatively large polydispersive efficiencies measured when emitting EMI- $\mathrm{BF}_{4}$ are consistent with emissions within or close to the $\operatorname{PIR}[7,16,41]$. Conversely the larger spread in constituent masses and possible droplet tail, after removing offsets, observed from both EMI-Im sources contributed to lower efficiency. For those devices, the negative polarity mass flow rate, proportional to the factor $\phi$, was roughly double that at positive potential. Within the standard deviations quoted, all parameters measured from both devices emitting EMI-Im were consistent.

\subsubsection{Angular Current Distribution}

Figure 14 provides examples of the collected current to emitted current fraction versus thruster angle with the axis of rotation orientated parallel and perpendicular to the emission strips. Parallel rotations were recorded from all devices, while perpendicular rotations were only measured from SRC-Im-1 and SRC-Im-2. The prevalence of multiple Taylor cones (emission sites) per emitter structure in porous ILIS in [24] has driven the development of the linear emission strip geometry. However; that same work found that emission sites are expected wherever the field is strong enough and may, as a result, not be well aligned axially. In this context, the reasonably well collimated beams shown are a welcome confirmation that conventional machining can produce sufficiently sharp structures to avoid wide beam angles.

For all devices, more current relative to that emitted was collected in the negative polarity versus positive. As shown by the indicated insets in Figures 14(a) and 14(b), the two polarities nevertheless are in agreement when scaled by their respective maxima. Considering the five $81 \%$ geometric transparency grids between the source and detector and the finite source dimensions, the negative current was typically consistent with sampling the entire beam with a source misalignment of a few $\mathrm{mm}$. The reduction in positive current may be attributed to neutralization by secondary electrons born from the impact of ions on the baffles and aperture plate surrounding the narrow ToF tube inlet aperture.

An axisymmetric approximation was made from each set of data bi-directional rotation data, facilitating use of the right hand side of equation 12. This process in- 
TABLE IV: Summary of Indirect Propulsive Parameters*

\begin{tabular}{|c|c|c|c|c|c|c|c|c|}
\hline Device & Polarity & $\kappa$ & $\phi$ & $\frac{\kappa^{2}}{\phi}(\%)$ & $\left\langle\frac{\Delta V_{\mathrm{ToF}}}{V_{e m}}\right\rangle(\%)$ & $\left\langle\frac{\Delta V_{\mathrm{RPA}}}{V_{e m}}\right\rangle(\%)$ & $\theta_{0}$ & $\theta_{c}$ \\
\hline \multirow[t]{4}{*}{ SRC-Im-1 } & + & $1.90 \pm 0.06$ & $5.1 \pm 0.7$ & $70 \pm 11$ & $2.1 \pm 1.0$ & $2.2 \pm 0.9$ & $\| 0.1^{\circ}$ & $\| 21.3^{\circ}$ \\
\hline & & & & & & & $\perp 2.0^{\circ}$ & $\perp 20.0^{\circ}$ \\
\hline & - & $2.60 \pm 0.18$ & $9.8 \pm 2.4$ & $69 \pm 19$ & $0.9 \pm 0.2$ & - & $\| 0.2^{\circ}$ & $\| 22.4^{\circ}$ \\
\hline & & & & & & & $\perp 2.8^{\circ}$ & $\perp 21.2^{\circ}$ \\
\hline \multirow[t]{4}{*}{ SRC-Im-2 } & + & $1.83 \pm 0.06$ & $4.8 \pm 0.7$ & $70 \pm 12$ & $1.8 \pm 0.8$ & $3.0 \pm 0.8$ & $\| 4.5^{\circ}$ & $\| 21.3^{\circ}$ \\
\hline & & & & & & & $\perp 0.5^{\circ}$ & $\perp 21.7^{\circ}$ \\
\hline & - & $2.71 \pm 0.19$ & $11.7 \pm 2.7$ & $63 \pm 17$ & $0.9 \pm 0.2$ & - & $\| 5.7^{\circ}$ & $\| 21.6^{\circ}$ \\
\hline & & & & & & & $\perp 1.7^{\circ}$ & $\perp 22.0^{\circ}$ \\
\hline \multirow[t]{2}{*}{$\mathrm{SRC}-\mathrm{BF}_{4}$} & + & $1.48 \pm 0.02$ & $2.6 \pm 0.3$ & $83 \pm 9$ & $6.7 \pm 1.3$ & - & $\| 5.5^{\circ}$ & $\| 20.8^{\circ}$ \\
\hline & - & $1.45 \pm 0.03$ & $2.7 \pm 0.3$ & $77 \pm 9$ & $3.5 \pm 0.4$ & - & $\| 3.4^{\circ}$ & $\| 20.2^{\circ}$ \\
\hline
\end{tabular}

*Uncertainties indicate the standard deviation over 19 or more measurements

cluded determining an offset angle $\left(\theta_{0}\right)$, taken as that angle about which axisymmetric surface integrations using relatively positive or negative rotation angles are equal. These two halves were then averaged to yield a single representative profile for each orientation and/or voltage.

Given the inconsistency between the integrated and expected beam currents, axisymmetric approximations for each device/polarity have been normalized such that the integration of $\bar{j}(\theta)$ over $S$ is unity. The effective beam angle has then been calculated by equation 12 , see table IV. In the table, $\|$ and $\perp$ indicated rotations parallel and perpendicular to the emitter strips respectively. Measurements repeated at differing emitter voltages were consistent to within $\sim 2^{o}$. The effective cone angle $\theta_{\text {eff }}$ was close to $21^{\circ}$ for all cases, corresponding to a loss of $\sim 7 \%$ in thrust output compared with a perfectly collimated beam. The consistency in $\theta_{\text {eff }}$ between rotation orientations, despite a more narrow profile in Figure 14(b) versus 14(a), reflects the diminishing returns, in terms of propulsive performance, achieved when collimating the central portion of the beam. Rather the small current contributions beyond $\sim 35^{\circ}$, in both orientations, are a relatively significant inefficiency driver.

\subsubsection{Energy Spectra}

RPA data were limited to constant positive $V_{e m}$ and zero rotation angle. The data, see Figure 15, confirm a primary population of particles had energies close to that of the emitters. However, a significant portion of the collected beam was, apparently, repelled at a lower energy. In Ref. [34] and [36] low energy beam repulsion was attributed to downstream ion fragmentation. However, downstream fragmentation manifests as a relatively discrete step/peak at a known energy corresponding to breakup in a field free region. The expected peak locations due to such events, are readily determined by scaling the initial particle energy $q V_{e m}$ by the ratio of post to pre breakup ion mass[34]. Specifically, if a solvated ion of mass $m_{n}$ has kinetic energy $q V_{e m}$ and breaks into a charged particle of mass $m_{n-1}$ and a neutral of mass $m_{n}-m_{n-1}$ the charged particle (ion) will have energy $K_{n-1}$ given by equation 16 .

$$
K_{n-1}=\frac{m_{n-1}}{m_{n}} q V_{e m}
$$

Emitting positive EMI-Im ions, the post-fragmentation ion energies will then be only $22 \%$ and $56 \%$ of the initial kinetic energy after $n=1 \rightarrow 0$ and $n=2 \rightarrow 1$ transitions respectively. These energy levels are indicated in Figure 15(a). Although the $n=1 \rightarrow 0$ marker did tend to indicate the lower limit of species energy, prominent peaks were not observed. In addition to breakup within the emitter to extractor accelerator region as discussed in the context of ToF measurements above, these measurements could therefore indicate that significant fragmentation occurred within the RPA's deceleration field. Compared with[34], the RPA setup here did not include an upstream Einzel lens and was therefore positioned closer to the source. Ultimately, the physical extent of the multiple retarding grids was roughly equal to the flight distance prior to the RPA. Hence, relatively little flight distance/time was available for breakup to occur downstream of the extractor grid yet prior to entering the probe.

The peak energy deficit was found to be typically within 20 to $80 \mathrm{~V}$ of $V_{e m}$ after correction for the current monitor resistance. These small deficits, a few percent of the emitter voltage, are in reasonable agreement with the average deficits estimated by ToF, see table IV. However; they are, again, large compared with previous studies of ILIS emitting EMI-Im. Specifically, Lozano[34] measured only a few $(\sim 5-7 \mathrm{~V})$ volt deficits and FWHM of similar order. Here the FWHM was typically $\sim 10$ $40 \mathrm{~V}$. The same instructions, Ref [47], were applied to our RPA and that of Lozano. Hence we anticipated a similar high resolution probe however, it should be noted that the resolution of our RPA was not rigorously verified with a monoenergetic reference source. 


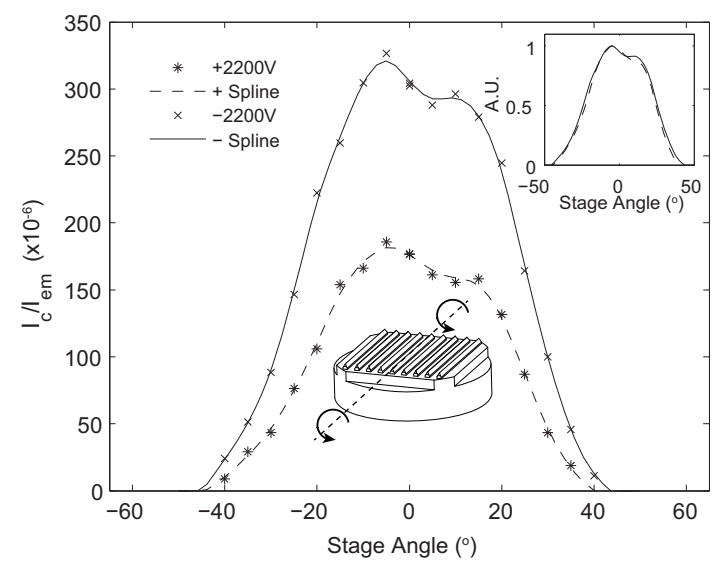

(a)Rotation parallel to the strip edges.

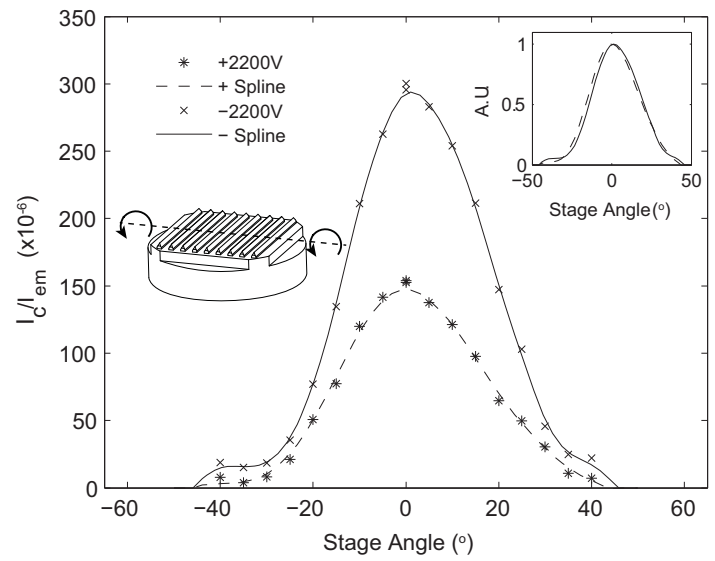

(b)Rotation perpendicular to the strip edges.

FIG. 14: Beam current fractions versus angle when rotating on the indicated axes. The inset figures demonstrate that, when normalized to the peak collection ratio, the positive and negative data are consistent.

\section{DISCUSSION}

\subsection{Comparison of Direct and Indirect Thrust Measurements}

Direct (DTM) and indirect (ITM) thrust measurements are plotted for each of the three tested devices in Figures 16 and 17. The ITM correspond to $T_{\mathrm{ITM}}=$ $\alpha T^{+}+(1-\alpha) T^{-}$where the thrusts $T^{ \pm}$for each polarity were determined through equation 13 using the indirect propulsive parameters listed in table IV and the factor $I_{b} \sqrt{\left|V_{e m}\right|}$ measured at each direct thrust measurement. Averaged input powers are indicated in the figure insets. All devices generated 10's of $\mu N$ with less than $0.8 \mathrm{~W}$ of input power. Maximum thrust levels of $\sim 50 \mu N$ and $\sim 30 \mu N$ were measured when emitting the ILs EMI-Im and EMI-BF ${ }_{4}$ respectively. Higher thrusts from EMI-Im were anticipated due to the higher anion mass.

The directly measured and indirectly calculated thrusts from SRC-Im-2 and SRC-BF 4 were in good agreement for all but the highest emission levels. Specifically,

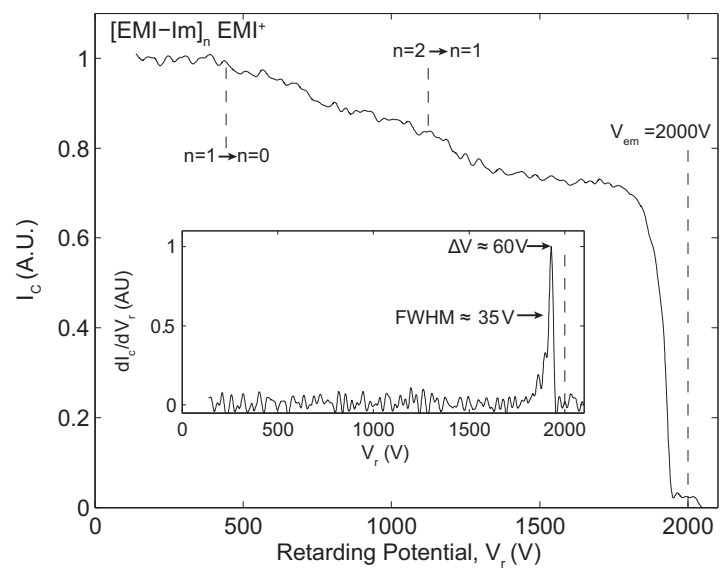

(a)SRC-Im-1

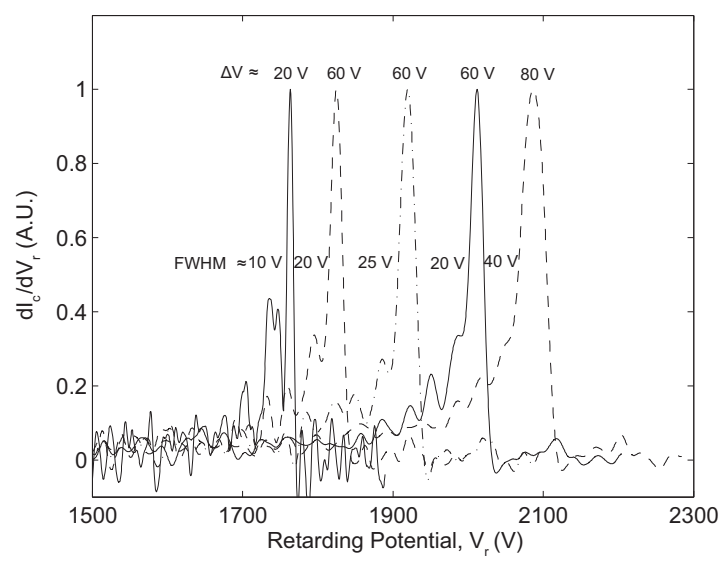

(b)SRC-Im-2

FIG. 15: (a) Smoothed current data measured against the retarding potential from SRC-Im-1. Vertical lines indicate the anticipated location of peaks associated with downstream (of the extractor) solvated ion breakup. (b) Distributions of current over retarding potential from SRC-Im-2. Peak energy deficits, with respect to $V_{e m}$ and FWHMs were typically several 10's of $V$.

the values agreed to within a few $\mu N$ up to $\sim 40 \mu N$ and $\sim 20 \mu N$ for SRC-Im-2 and SRC-BF 4 respectively, with deviations closer to $5 \mu N$ at higher thrust levels. Less consistent agreement was observed from SRC-Im-1 where disparities approached $15 \mu N$ at high power.

The predicted thrusts tended to exceed that emitted at high current. The composition, angle and energy thrust coefficients have been approximated as constant averages over all voltages and angles. We therefore only consider the bulk standard deviations in $\kappa$ listed in table IV, which correspond to up to $\sim 3 \mu N$, as providing bounds to the calculations. Were more consistent ToF measurements obtained, the divergence at high voltage may have been reduced through parameterizing $\kappa$ versus voltage. Regardless, lower than expected thrusts are supportive of highly ionic emissions since an undetected droplet population would have lead to a higher thrust.

Inadequate thrust balancing may have contributed to 


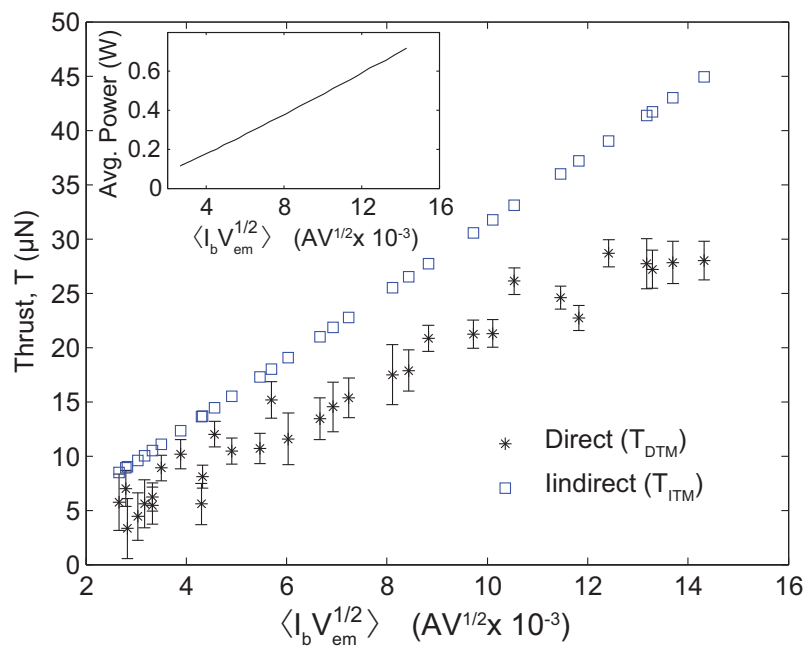

(a)SRC-Im-1

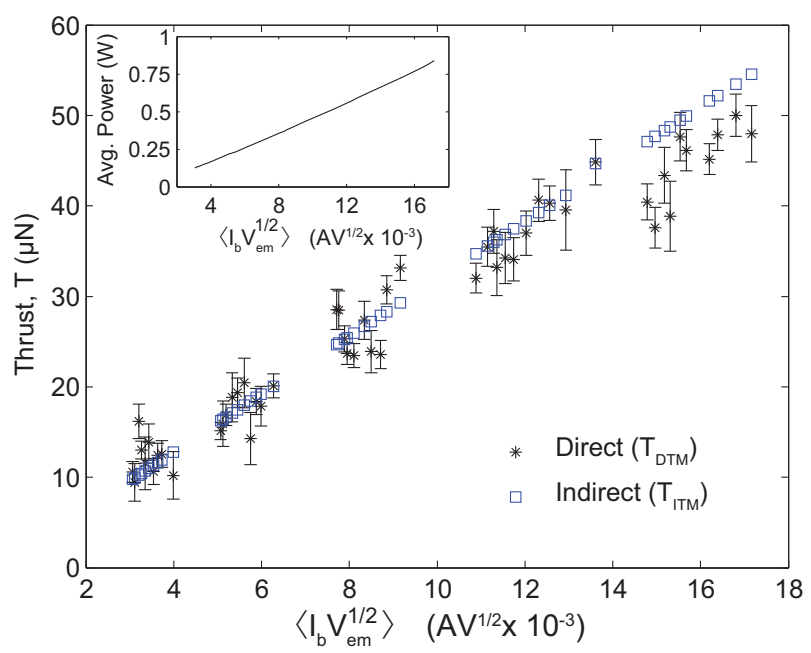

(b)SRC-Im-2

FIG. 16: Summary of the directly (DTM) and indirectly (ITM) measured thrusts from devices wet with EMI-Im. The indirect thrust measurement standard error was $\sim \pm 2-3 \mu N$. Excellent agreement between direct and indirect measurements was obtained for SRC-Im-2 at most setpoints.

the disagreement in Figure 16(a) for SRC-Im-1. Referring to table IV, the ratio of positive to negative $\kappa$ parameters calculated by ToF was approximately $\sim 0.7$ for both SRC-Im- 1 and SRC-Im-2, and $\sim 1.0$ for device SRC-BF 4 . The ratios applied to establish DTM setpoints, table II, were similar for SRC-Im-2 and SRC-BF 4 yet SRC-Im-1 may have been relatively poorly balanced. In all cases, force imbalances have been accounted for in $T_{\mathrm{ITM}}$ to a degree through the duty cycle weighted average described; however, the response of the balance to pulsations, as may have been present particularly with SRC-Im-1, was unknown.

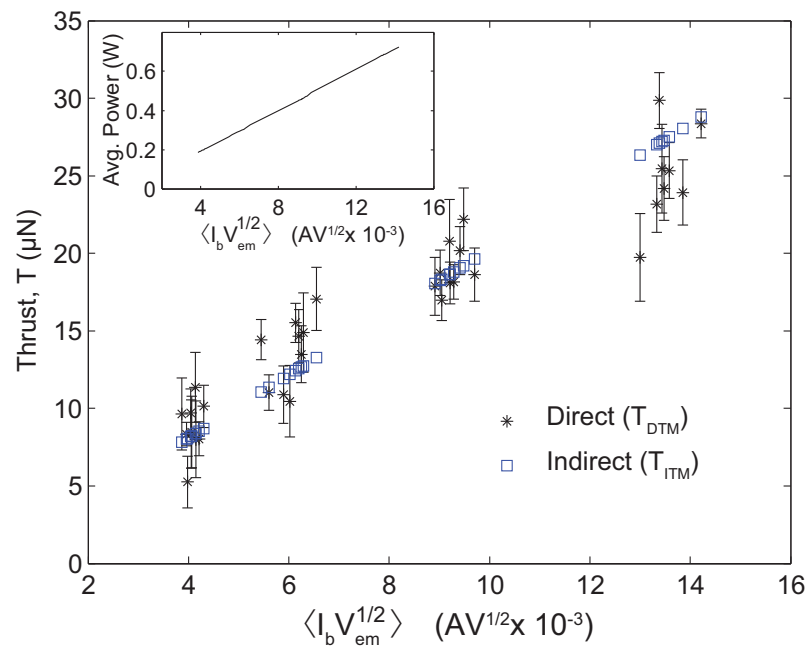

FIG. 17: Agreement between directly (DTM) and indirectly (ITM) measured thrusts from $\mathrm{SRC}_{-} \mathrm{BF}_{4}$. The indirect thrust measurement standard error was $\sim \pm 1 \mu N$.

\subsection{Compiled Thruster Performance}

The agreements between direct and indirect thrust measurements from the same devices, a unique scenario for a passively fed and ionic electrospray thruster, permit total performance estimates. In table $\mathrm{V}$ we have interpolated the propulsive efficiency, specific impulse and thrust at $0.5 \mathrm{~W}$ of input power. The specific impulse and propulsive efficiency have been calculated by equations 14 and 15, modified to account for potential alternation. For all devices, values in parentheses were obtained when using an interpolation of the directly measured thrust; although the calculated mass flow was used for all values. In this form the data re-affirm the improved specific performance of EMI-BF 4 over EMI-Im, at the expense of reduced thrust, due to greater ion content and lower anion mass associated with EMI-BF ${ }_{4}$ emissions. Given the comprehensive nature of the diagnostic measurements presented, and good agreement with direct measurements for two of three device, we are confident that these performance metrics present an accurate portrayal of the capabilities available from such a device.

In interpreting these data, the implicit scalability of ILIS thrusters should be considered. The active area of these prototype devices was roughly $1 \mathrm{~cm}^{2}$; hence numerous sources could be accommodated within a small area thereby increasing the thrust. Indeed, to obtain charge neutrality, the intended application would include at least two sources operated simultaneously at opposite polarities.

Referring to equation 15 and table IV it is clear that polydispersive efficiency represents the greatest inefficiency within the system; consistent with the efforts to increase this parameter discussed in the introduction. Beam spreading and interception by the extrac- 
TABLE V: Thruster Performance Summary at $0.5 \mathrm{~W}$

\begin{tabular}{llcc}
\hline \hline Device & $\mathrm{T}(\mu N)$ & $I_{s p}(s)$ & $\eta_{\text {prop. }}(\%)$ \\
\hline SRC-Im-1 & $33(23)$ & $1660(1120)$ & $53(25)$ \\
SRC-Im-2 & $35(34)$ & $1440(1410)$ & $49(47)$ \\
SRC-BF $_{4}$ & $20(20)$ & $3260(3290)$ & $65(66)$ \\
\hline \hline
\end{tabular}

() indicate values calculated by interpolation of DTM.

tion grid were the next most significant contributors respectively; although for $\mathrm{SRC}_{-} \mathrm{BF}_{4}$ energy deficit losses also approached $7 \%$. Grid interception must be reduced through design improvements, not only to improve performance but to eliminate erosion as a lifetime limitation. Meanwhile beam spread over angle would be improved to a degree if a downstream secondary accelerating electrode were included[26]; although the efficiency benefits would likely be small. The higher energy deficits compared with the literature should be further explored. The ill defined IL to aluminum contact made through the guard ring may have contributed to this loss, although the deficits were only weakly correlated with $V_{e m}$ (and therefore $I_{e m}$ ) indicating loss mechanisms were not entirely Ohmic. Despite these losses, when compared with other miniaturized ion propulsion systems[11] and considering the system benefits of completely passive (un-powered) propellant feeding, the high specific impulses and $\sim 50 \%$ to 65 $\%$ power efficiencies achieved with these prototypes are encouraging.

\subsection{Emission Decay and Longevity}

This initial study has been intended to confirm the efficacy of indirect thrust measurements and to demonstrate that a simple fabrication process can be used to form high current porous emission sources. Many design improvements are required to transition from these demonstration thrusters to engineering breadboards suitable for long duration tests and precise thrust resolution measurements. Decays in emission current over the course of

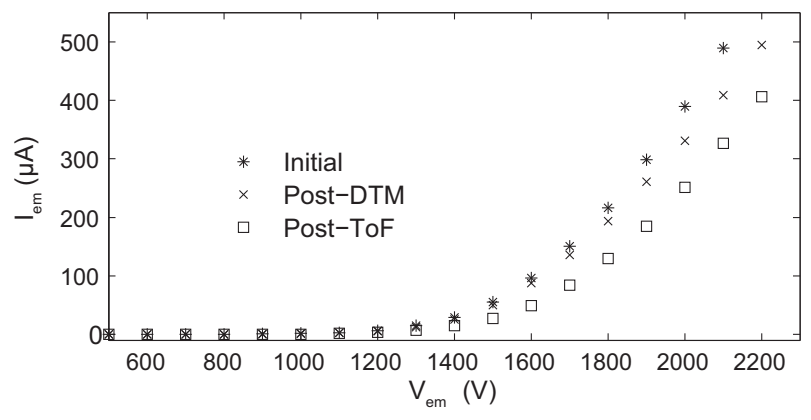

FIG. 18: Emission currents tended to decay over the course of measurements, particularly after ToF measurements which necessitated prolonged constant polarity emissions. the test campaign were a particular concern. Figure 18 provides an example of the decrease in emitted current before and after direct thrust measurements at ESTEC and subsequently after ToF measurements at EPFL. It is tempting to broadly attribute this behavior to degradation due to electrochemical reactions at the ionic liquid to guard ring contact interface[2, 44]. We have followed the most recent recommendations in the literature whenever possible: use of a the glass emitter substrate enforces a distal electrical contact[46] and attempts were made to balance positive and negative emission charge transfer over each period of potential alternation during thrust measurements[33, 50]. However; long (>10 s) periods of sustained unipolar operation were unavoidable with the ToF and RPA systems as configured, and the efficacy of potential alternation to suppress reactions was not confirmed within our specific application. If, for example, reactions were occurring at the ill-defined aluminum to IL interface the effective current path resistance could have been increasing, despite the absence of breakdowns at the glass emission sites. To gain further insight into
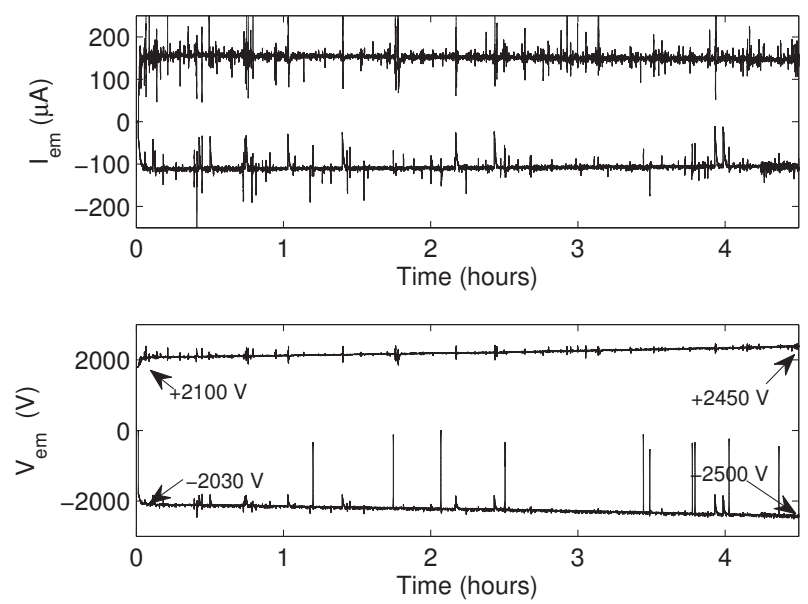

FIG. 19: One device was run for 4.5 hours with a closed loop feedback control set to maintain $15 \mu N$ of predicted thrust by maintaining a prescribed $\left\langle I_{b} \sqrt{\left|V_{e m}\right|}\right\rangle$. As the current decayed, the voltage increased accordingly.

emission decays we operated SRC-Im-1 continuously for $>4.5$ hours at $1 \mathrm{~Hz}$ and with a closed loop feedback mechanism to ensure constant charge balance while also attempting to maintain $20 \mu N$ of predicted thrust output in each polarity through constant $\pm I_{b} \sqrt{\left|V_{e m}\right|}$ setpoints. The current and voltage in each polarity are presented in Figure 19 over the course of the test. The voltage required to sustain the commanded thrust rose steadily from $+2100 /-2030 \mathrm{~V}$ to $+2450 /-2500 \mathrm{~V}$ while the current decayed accordingly. It is notable that the average power rose less dramatically, from $\sim 0.29 \mathrm{~W}$ to $\sim 0.32 \mathrm{~W}$. The current spikes visible were, in part, indications of deficiencies in the simple controller employed which could 
not rapidly suppress surges.

The test was ended intentionally, no specific or dramatic failure mode was encountered and liquid remained in the device. The total time required to deplete the available propellant was calculated using equation 7 with the coefficients $\phi$ listed in table IV and the average currents measured during this test. If the device were freshly loaded with the initial 110 to $120 \mu \mathrm{L}$ of EMI-Im, all liquid would have been consumed after approximately 40 hours. In actuality, the device had already been operated for approximately 2 hours during the thrust and diagnostic data acquisitions prior to beginning this test. However; even considering this time, many more hours would have been required to exhaust all available propellant.

At the culmination of the test we inspected the device. Most evidently, the extractor grids were eroded near the emitter strip ends and the previously white/clear surface was distinctly yellow. Erosion was not unexpected at these positions since the emitting strips terminate abruptly at each end, resulting in sharp features perpendicular to the desired orientation. Emissions from these ends had struck the extractor grid and, after several hours, caused significant erosion. The accompanying sputtered material may also be responsible for some of the observed discoloration and, possibly, current decay through contamination of the emitting surfaces.

\subsection{Triangular Prisms as Emission Sources}

The conventional machining approach applied was successful in obtaining up to $500 \mu \mathrm{A}$ of highly ionic emission from $1 \mathrm{~cm}$ diameter active areas; current densities similar to reported measurements from 100's of axisymmetric porous emitters multiplexed in arrays $[2,5]$. The number of active emission sites was not confirmed directly. Maximum reported currents from individual axisymmetric porous emitters have ranged from 100's of $n A[5]$ to more than $5 \mu A[23]$. The hundreds of $\mu A$ reported here are therefore broadly indicative of activating several hundred emission sites over the 9 emitting edges. However, the number of sites per structure in the literature has not been reported with certainty. Furthermore, a definitive or phenomenological maximum current level was not reached here nor has one been typically reported from previously reported devices using structures with a porous bulk material. Correspondingly, detailed quantitative comparisons between current levels and emitter densities in the literature as means to calculate emission site distributions are imprudent at present.

As local field strengths will have exceeded the minimum threshold for emission at differing voltages along the undulated edges, the number of active sites likely increased with voltage. Further study is required to establish criteria for emission site spacing; accounting for potential hydrodynamic interactions and space charge effects along with the local electrostatics. Such an understanding will be important in developing the design.

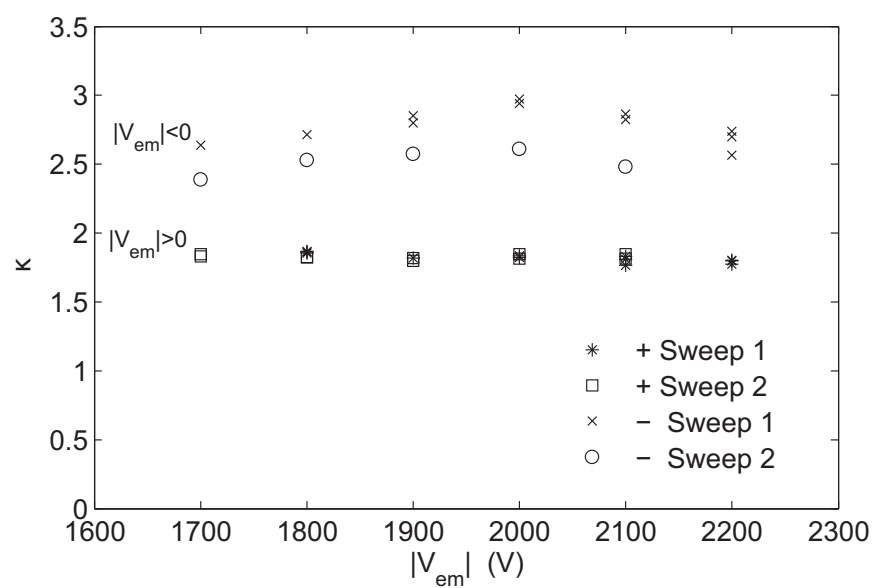

(a)

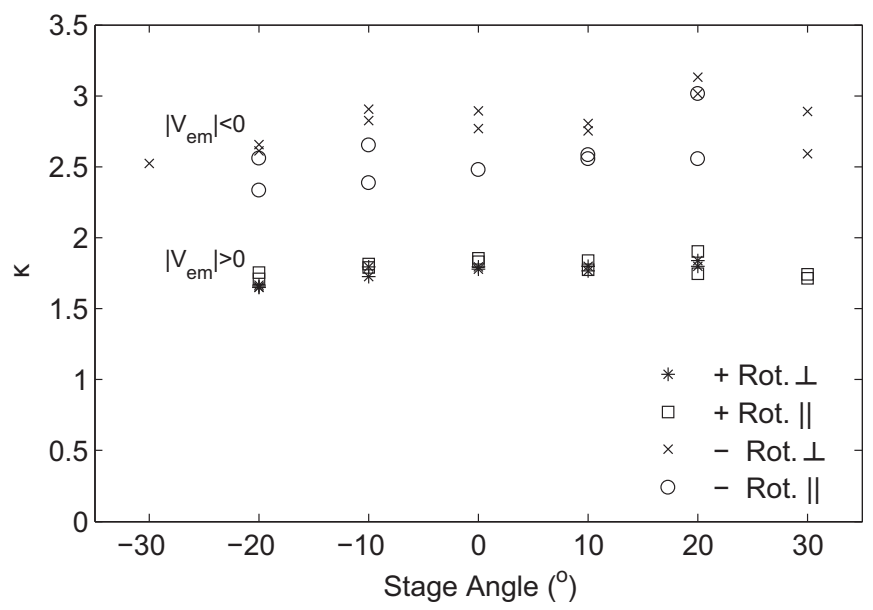

(b)

FIG. 20: Small and inconsistent trends were observed when measuring the ToF paramater $\kappa$ at differing voltages (a) and beam angles (b). In (a) sweeps indicate repeated data acquisition under the same conditions.

For example, the emitting edges protruded into the grid thickness for both EMI-Im devices. Some emissions from sites off the symmetry axis of those devices may have been lost due to strong electric fields oriented towards the grid walls. As with misguided emissions from the ends of each strip, resultant sputtered material or secondary electrons could have interacted with the nearby IL and may have contributed to the degrading emission over time. Enhanced knowledge of emission site localization may also be beneficial to interpreting fragmentation events through providing an improved understanding of the electric field each particle experiences after emission.

ToF measurements were made at differing emitter voltages and beam angles. However; all measurements were averaged to provide single propulsive metrics for each device and polarity. This decision was made for two reasons. Firstly, the beam current decays over the course of ToF measurements resulted in ambiguity when attempting to attribute voltage resolved ToF parameters with 
the conditions present during DTM. Second, any changes in propulsive metrics which were observed tended to be relatively small and comparable to the deviations inherent when repeating measurements at consistent inputs. Specifically, no clear trends were visible in beam composition versus extraction voltage nor angle, via either the thrust metric $\kappa$ nor the mass flow rate factor $\phi$. For example, figures 20(a) and 20(b) demonstrate the calculated parameter $\kappa$ versus voltage (at $0^{\circ}$ ) and versus angle respectively from SRC-Im-2.

The lack of trend versus beam angle result would be particularly noteworthy for an individual emitter or an array of geometrically identical emitters. Previous studies have, for example, found significant trends in mass flow rate (droplet content) versus angle from individual ILIS[37]. However; measurements versus angle here comprise a summation of currents due to beamlets originating at different surface angles and with differing intensities due to the lack of enforced emission site localization on the porous surface.

\section{CONCLUSION}

We have presented a simple to fabricate ionic electrospray thruster with fully passive propellant feeding. A small yet conventional CNC mill was used to machine triangular prisms, $\sim 300 \mu \mathrm{m}$ tall and with an apex radius of curvature of roughly $10-30 \mu \mathrm{m}$, from commercially available porous borosilicate filter discs. Three prototype thrusters have been tested, two emitting the ionic liquid EMI-Im and one using EMI-BF 4 . For all sources, several hundred $\mu A$ were emitted from less than $1 \mathrm{~cm}^{2}$ of active area at voltages near $2000 \mathrm{~V}$. Despite limited control over the emitting apex curvature, the emission spread over angle was equivalent to $\mathrm{a} \sim 21^{\circ}$ hollow cone, representing a small $\sim 7 \%$ reduction in thrust over a perfectly collimated beam.

The utility of such a source as a compact thruster has been demonstrated through direct thrust measurements. Controllable thrusts from $5 \mu N$ to $50 \mu N$, corresponding to $0.1 W$ to $0.8 W$, were recorded when emitting EMIIm and from $7 \mu N$ to $25 \mu N$ at $0.2 W$ to $0.7 W$ when emitting EMI-BF 4 . These data were measured while alternating the emission polarity at $1 \mathrm{~Hz}$ while attempting to balance total charge emission through appropriate selection of the duty cycle; in an effort to suppress electrochemical degradation. The thrusters were controlled by an applied emitter to extractor grid voltage alone, propellant IL was passively drawn, by capillarity, from an integral porous reservoir.

Accurate calculation of thrust using indirect probe measurements has been discussed and demonstrated. Through defining non-dimensional modifiers describing the average mass to charge ratio of constituent beam particles, an effective beam angle and energy deficits compared with the applied voltage we have made calcula- tions of thrust output as a function of beam current and emission voltage. For two of three devices, these calculations matched the directly measured thrust to within a few $\mu N$. Specifically, we have shown that while Time of Flight spectrometry alone can provide a thrust estimate close to that directly measured, modifications due to beam angle and energy deficits should be included to obtain good accuracy. The effects of fragmentation within the emitter to extractor grid were also considered yet were found to have a small $(\sim 3 \%)$ influence on thrust.

The mass flow rate has been similarly calculated using ToF measurements and the results have been applied, along with thrust, to calculate the expected specific impulse and total propulsive efficiencies of the prototype thrusters. Emissions from EMI-Im included a small droplet, or highly solvated ion, content; representing up to $10 \%$ of the total current during negative polarity operation. This population contributed to relatively low calculated propulsive efficiencies and specific impulses, roughly $50 \%$ and $1500 s$ at $0.5 \mathrm{~W}$ from devices emitting EMI-Im. Conversely, spectra from EMI-BF ${ }_{4}$ emissions revealed a lower intensity $(<4.5 \%)$ and lower mass solvated ion tail; consistent with this liquid approaching the PIR. Consequently, higher propulsive efficiency (65 $\%)$ and specific impulse (3260 s) were calculated.

These novel, conventionally machined thrusters can enable low-cost and accessible study of the operation of porous IL electrospray sources without an emphasis on device fabrication; beginning with this study of thrust and propulsive performance. We intend to continue these investigations through i) developing the device design, in order to increase the operational lifetime and improve emission consistency, and ii) continued efforts to seek experimental evidence of the predicted performance benefits of ILIS thrusters. The latter will include measurements of thrust output while simultaneously emitting balanced currents, of opposite polarity, in order to confirm charge neutralization and measurements of true specific impulse by directly recording the propellant mass reduction over very long durations while monitoring thrust.

\section{Acknowledgments}

This work has been supported through ESA NPI contract \#4000109063/13/NL/PA. The authors would like to thank Alexandra Bulit, Davina Di Cara, Kathe Dannenmayer and Eduard Bosch Borras of the ESA Propulsion Laboratory for their assistance with the direct thrust measurements.

\section{References}


[1] Gassend, B., Velasquez-Garcia, L. G., M., A. I. A., and Martinez-Sanchez, "A microfabricated planar electrospray array ionic liquid ion source with integrated extractor," Microelectromechanical Systems, Journal of, Vol. 18, No. 3, 2009, pp. 679-694.

[2] Courtney, D. G., Li, H. Q., and Lozano, P., "Emission Measurements from Planar Arrays of Porous Ionic Liquid Ion Sources," IOP Journal of Physics D, Vol. 45, No. 48, 2012, p. 485203.

[3] Stark, J., Stevens, B., Alexander, M., and Kent, B., "Fabrication and Operation of Microfabricated Emitters as Components for a Colloid Thruster," Journal of Spacecraft and Rockets, Vol. 42, No. 4, 2005, pp. 628-639.

[4] Krpoun, R. and Shea, H. R., "Integrated out-of-plane nanoelectrospray thruster arrays for spacecraft propulsion," Journal of Micromechanics and Microengineering, Vol. 19.

[5] Coffman, C., Perna, L., Li, H., and Lozano, P. C., "On the Manufacturing and Emission Characteristics of a Novel Borosilicate Electrospray Source," in " $49^{\text {th }}$ AIAA Joint Propulsion Conference and Exhibit," San Jose, CA, 2013.

[6] Romero-Sanz, I., Bocanegra, R., Gamero-Castan̆o, M., de la Mora, J. F., and Lozano, P., "Source of heavy molecular ions based on Taylor cones of ionic liquids operating in the pure ion evaporation regime," Journal of Applied Physics, Vol. 94, No. 5, 2003, pp. 3599-3605.

[7] Lozano, P. and Martínez-Sánchez, M., "Ionic liquid ion sources: characterization of externally wetted emitters," Journal of Colloid and Interface Sciences, Vol. 282, No. 2, 2005, p. 415.

[8] Lozano, P. and Martínez-Sánchez, M., "Efficiency Estimation of EMI-BF 4 Ionic Liquid Electrospray Thrusters," in " $41^{\text {st }}$ Joint Propulsion Conference \& Exhibit," Tuscon, Arizona, 2005.

[9] Taylor, G. I., "Disintegration of Water Drops in an Electric Field," Proceedings of the Royal Society of London A, Vol. 280, No. 1382, 1964, pp. 383-397.

[10] Warner, N. Z., "Theoretical and Experimental Investigation of Hall Thruster Miniaturization," Ph.D. Thesis, Massachusetts Institute of Technology, Cambridge, MA, 2007.

[11] Mueller, J., Hofer, R., and Ziemer, J., "Survey of propulsion technologies applicable to cubesats," NASA Technical Report, , No. 10-1646.

[12] Dandavino, S., "Microfabricated Electrospray Thrusters for a Modular Spacecraft Propulsion System," Ph.D. Thesis, Ecole Polytechnique Federale de Lausanne, 2014.

[13] Ziemer, J. K. and Merkowitz, S. M., "Microthrust Propulsion for the LISA Mission," in " $40^{\text {th }}$ Joint Propulsion Conference," Fort Lauderdale, FL, 2004. Also AIAA-2004-3439.

[14] Marcuccio, S., Giusti, N., and Pergola, P., "Slit FEEP Thruster Performance with Ionic Liquid Propellant," in " $49^{\text {th }}$ AIAA Joint Propulsion Conference and Exhibit," San Josa, CA, 2013.

[15] Tajmar, M., Vasiljevich, I., Plesescu, F., Grienauer, W., Buldrini, N., Betto, M., and del Amo, J. G., "Development of a Porous Tungsten mN-FEEP Thruster," in "Space Propulsion 2010," San Sebastian, Spain, 2010.

[16] Legge, R. S. and Lozano, P. C., "Electrospray propul- sion based on emitters microfabricated in porous metals," Journal of Propulsion and Power, Vol. 27, No. 2, 2011, pp. 485-495.

[17] Hill, F. A., Leon, P. P. D., and Velásquez-García, L. F., "High-throughput ionic liquid electrospray sources based on dense monolithic arrays of emitters with integrated extractor grid and carbon nanotube flow control structures," in " $17^{\text {th }}$ International Conference on SolidState Sensors, Actuators and Microsystems, Transducers 2013," IEEE, 2013, pp. 2644-2647.

[18] Ziemer, J. K., Randolph, T. M., Franklin, G. W., Hruby, V., Spence, D., Demmons, N., Roy, T., Ehrbar, E., Zwahlen, J., Martin, R., and Connolly, W., "Colloid micro-newton thrusters for the space technology 7 mission," in "IEEE Aerospace Conference 2010," Big Sky, MT, 2010. Also AERO-2010-5446760.

[19] Gamero-Castan̆o, M. and Hruby, V., "Electrospray as a Source of Nanoparticles for Efficient Colloid Thrusters," Journal of Propulsion and Power, Vol. 17, No. 5, 2001, pp. $977-987$.

[20] Alonso-Matilla, R., Fernández-García, J., Congdon, H., and de la Mora, J. F., "Search for liquids electrospraying the smallest possible nanodrops in vacuo," Journal of Applied Physics, Vol. 116, 2014, p. 224504.

[21] Lenguito, G. and Gomez, A., "Development of a multiplexed electrospray micro-thruster with post-acceleration and beam containment," Journal of Applied Physics, Vol. 114, 2013, p. 154901.

[22] Ryan, C., Daykin-Iliopoulos, A., Stark, J. P. W., Salaverri, A., Vargas, E., Rangsten, P., Dandavino, S., Ataman, C., Chakraborty, S., Courtney, D., and Shea, H., "Experimental progress towards the MicroThrust MEMS electrospray electric propulsion system," in " $33^{\text {rd }}$ International Electric Propulsion Conference," Washington, DC, 2013. Also IEPC-2013-146.

[23] Courtney, D. G. and Lozano, P., "Characterization of Conical Ionic Liquid Ion Sources for 2-D Electrospray Thruster Arrays on Porous Substrates," Transactions of the Japan Society for Aeronautical and Space Sciences, Aerospace Technology Japan, Vol. 8, 2010, pp. 73-78.

[24] Courtney, D. G., "Ionic Liquid Ion Source Emitter Arrays Fabricated on Bulk Porous Substrates for Spacecraft Propulsion," Ph.D Thesis, Massachusetts Institute of Technology, Cambridge, MA, 2011.

[25] Deng, W., Klemic, J. F., Li, X., Reed, M. A., and Gomez, A., "Increase of electrospray througput using multiplexed microfabricated sources for the scalable generation of monodisperise droplets," Aerosol Science, Vol. 37, 2006, pp. 696-714.

[26] Dandavino, S., Ataman, C., Ryan, C. N., Chakraborty, S., Courtney, D. G., Stark, J. P. W., and Shea, H., "Microfabricated electrospray emitter arrays with integrated extractor and accelerator electrodes for the propulsion of small spacecraft," Jounal of Micromechanics and Microengineering, Vol. 24, No. 7, 2014, p. 075011.

[27] Courtney, D. G., Li, H. Q., and Lozano, P., "Electrochemical Micromachining on Porous Nickel for Arrays of Electrospray Ion Emitters," Journal Of Microelectrochemical Systems, Vol. 22, No. 2, 2013, pp. 471-482.

[28] Meyer, I. V., Edmond, J., and King, L. B., "Electrospray from an Ionic Liquid Ferrofluid utilizing the Rosensweig 
Instability," in " $49^{t} h$ Joint Propulsion Conferene and Exhibit," San Jose, CA, 2013. Also AIAA-2013-3823.

[29] Lozano, P., Martínez-Sánchez, M., and Lopez-Urdiales, J. M., "Electrospray emission from nonwetting flat dielectric surfaces," Journal of Colloid and Interface Sciences, Vol. 276, 2004, pp. 392-399.

[30] Perel, J., Mahoney, J. F., Moore, R. D., and Yahiku, A. Y., "Research and Development of a Charged-Particle Bipolar Thruster," AIAA Journal, Vol. 7, No. 3, 1969, pp. 507-511.

[31] Legge, R. and Lozano, P., "Performance of Heavy Ionic Liquids with Porous Metal Electropsray Emitters," in " $44^{\text {th }}$ Joint Propulsion Conference \& Exhibit, AIAA2008-5002," Hartford, CT, 2008.

[32] Courtney, D. G. and Shea, H., "Accounting for Fragmentation in Time of Flight Spectrometry Based Calculations of Ionic Electrospray Thruster Performance," Journal of Propulsion and Power. Submitted.

[33] Castro, S. and de la Mora, J. F., "Effect of tip curvature on ionic emission from Taylor cones of ionic liquids from externally wetted tungsten tips," Journal of Applied Physics, Vol. 105, 2009, p. 034903.

[34] Lozano, P., "Energy Properties of an EMI-Im ionic liquid ion source," Journal of Physics D: Applied Physics, Vol. 39, 2006, pp. 126-134.

[35] Chakraborty, S., Ataman, C., Dandavino, S., and Shea, H., "Microfabrication of an electrospray thruster for small spacecraft," Proceedings of POWERMEMS, pp. 25.

[36] Fedkiw, T. P. and Lozano, P. C., "Development and characterization of an iodine field emission ion source for focused ion beam applications," Journal of Vacuum Science and Technology B, Vol. 27, No. 6, 2009, pp. 26482653.

[37] Chiu, Y.-H., Gaeta, G., Levandier, D. J., Dressler, R. A., and Boatz, J. A., "Vacuum electrospray ionization study of the ionic liquid,[Emim][Im]," International Journal of Mass Spectrometry, Vol. 265, No. 2, 2007, pp. 146-158.

[38] Martínez-Sánchez, M., "MIT Course 16.522, Space Propulsion Notes," MIT Open Coursware, http://ocw.mit.edu.

[39] Ziemer, J. K., "Performance of Electrospray Thrusters," in " $31^{\text {st }}$ International Electric Propulsion Conference," Ann Arbor, MI, 2009. Also IEPC-2009-242.

[40] Lozano, P. and Martínez-Sánchez, M., "Studies on the Ion-Droplet Mixed Regime in Colloid Thrusters," Ph.D Thesis, Massachusetts Institute of Technology, Cambridge, MA, 2003.
[41] Krpoun, R., Smith, K. L., Stark, J. P. W., and Shea, H., "Tailoring the hydraulic impedance of out-of-plane micromachined electrospray sources with integrated electrodes," Applied Physics Letters, Vol. 94, No. 16, 2009 p. 163502.

[42] Khayms, V., "Advanced Propulsion for Microsatellites," Ph.D Thesis, Massachusetts Institute of Technology, Cambridge, MA, 2000.

[43] Larriba, C., Garoz, D., Bueno, C., Romero-Sanz, I., Castro, S., and de la Mora, J. F., "Taylor Cones of Ionic Liquids as Ion Sources: The Role of Electrical Conductivity and Surface Tension," in Brennecke, J. F., Rogers, R. D., and Seddon, K. R., eds., "Ionic Liquids: Not Just Solvents Anymore," Oxford University Press, New York, 2007.

[44] Lozano, P. and Martínez-Sánchez, M., "Ionic liquid ion sources: suppression of electrochemical reactions using voltage alternation," Journal of Colloid and Interface Sciences, Vol. 280, 2004, pp. 149-154.

[45] de la Mora, J. F., Berkel, G. J. V., Enke, C., Cole, R. B., Martinez-Sanchez, M., and Fenn, J. B., "Electrochemical processes in electrospray ionization mass spectrometry," Journal of Mass Spectrometry, Vol. 35, No. 8, 2000, pp. 939-952.

[46] Brikner, N. and Lozano, P. C., "The role of upstream distal electrodes in mitigating electrochemical degradation of ionic liquid ion sources," Applied Physics Letters, Vol. 101, No. 19, 2012, p. 193504.

[47] Enloe, C. L. and Shell, J. R., "Optimizing the energy resolution of planar retarding potential analyzers," Review of scientific instruments, Vol. 63, No. 2, 1992, pp. 1788-1791.

[48] Fedkiw, T. P., "Characterization of an Iodine-Based Ionic Liquid Ion Source and Studies on Ion Fragmentation," S.M. Thesis, Massachusetts Institute of Technology, Cambridge, MA, 2010.

[49] Prince, B. D., Tiruppathi, P., Bemish, R. J., Chiu, Y.-H., and Maginn, E. J., "Molecular Dynamics Simulations of 1-Ethyl-3-methylimidazolium Bis [(trifluoromethyl) sulfonyl] imide Clusters and Nanodrops," The Journal of Physical Chemistry A, Vol. 119, No. 2, 2015, pp. 352368.

[50] Coffman, C., Courtney, D. G., Hicks, F., Jamil, S., Li, H., and Lozano, P., "Progress Toward a Variable Specific Impulse Electrospray Propulsion System," in " $47^{\text {th }}$ Joint Propulsion Conference \& Exhibit," San Diego, CA, 2011. AIAA-2011-5591. 